\title{
Supply Chain Risk Network Management: A Bayesian Belief Network and Expected Utility Based Approach for Managing Supply Chain Risks
}

\author{
Abroon Qazi $^{\mathrm{a}, *}$, Alex Dickson ${ }^{\mathrm{b}}, \mathrm{John}_{\text {Quigley }}{ }^{\mathrm{a}}$, Barbara Gaudenzi $^{\mathrm{c}}$ \\ ${ }^{a}$ Department of Management Science, University of Strathclyde, William Duncan Building, 130 Rottenrow, Glasgow, G4 \\ OGE, Scotland \\ ${ }^{b}$ Department of Economics, University of Strathclyde, William Duncan Building, 130 Rottenrow, Glasgow, G4 0GE, \\ Scotland \\ ${ }^{c}$ Department of Business Administration, University of Verona, Via Cantarane 24, Verona, Italy
}

\footnotetext{
*Corresponding author

Email addresses: abroon.qazi@strath.ac.uk (Abroon Qazi), alex.dickson@strath.ac.uk (Alex Dickson), j.quigley@strath.ac.uk (John Quigley), barbara.gaudenzi@univr.it (Barbara Gaudenzi)
} 
Graphical Abstracts (for review)

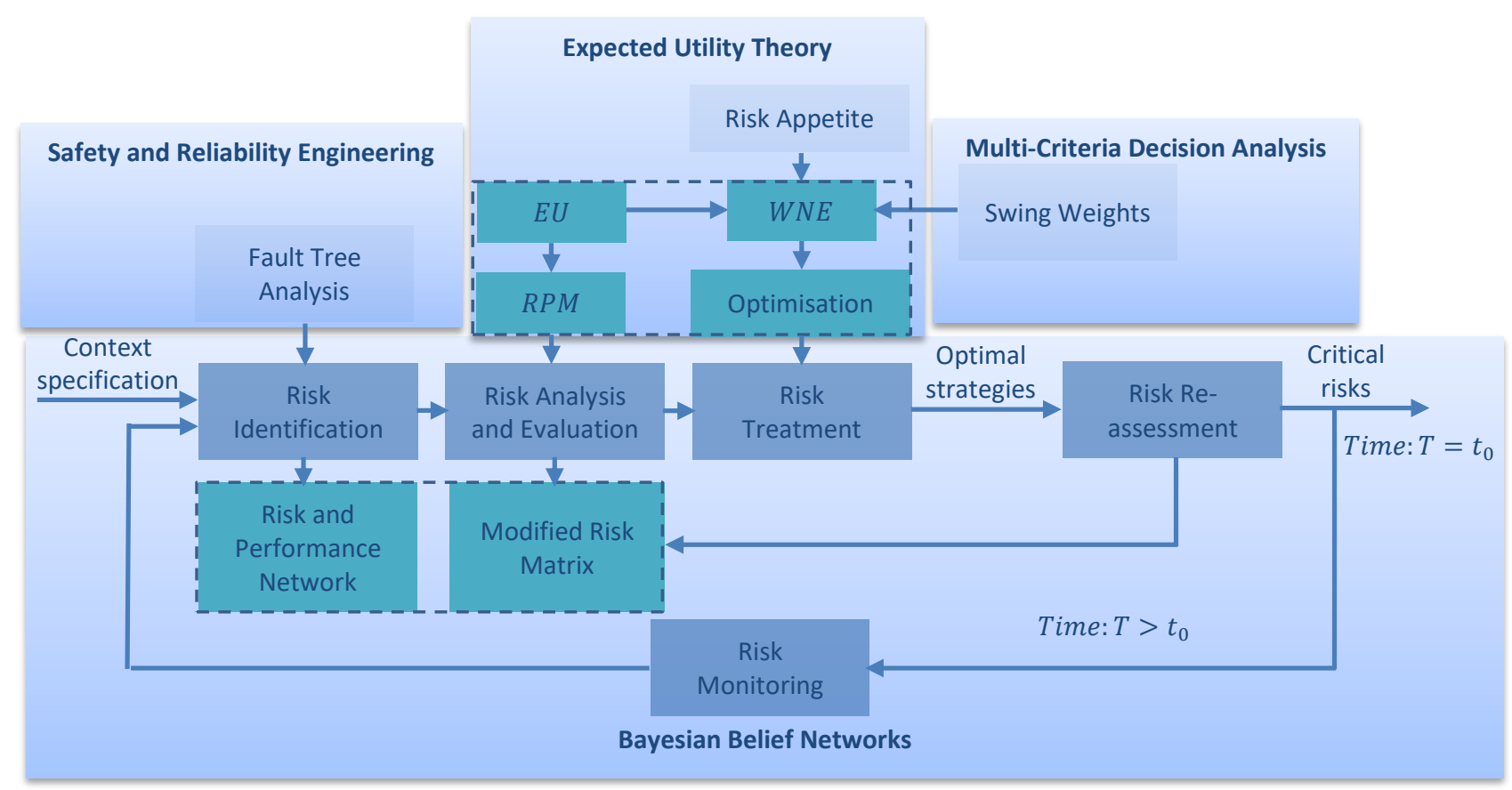


Highlights (for review)

\section{Highlights}

- A SCRM process integrating all stages of the risk management process is proposed and operationalised.

- The operationalisation scheme adapts and integrates various techniques from diversified research areas.

- 'Probability-conditional expected utility' matrix is introduced to assess interdependent risks.

- 'Weighted net evaluation of risk mitigation' is proposed to capture the decision maker's risk appetite.

- Propositions are introduced to elucidate the significance of modelling interdependent risks. 


\title{
Supply Chain Risk Network Management: A Bayesian Belief Network and Expected Utility Based Approach for Managing Supply Chain Risks
}

\begin{abstract}
The paper develops and operationalises a supply chain risk network management (SCRNM) process that captures interdependencies between risks, multiple (potentially conflicting) performance measures and risk mitigation strategies within a (risk) network setting. The process helps in prioritising risks and strategies specific to the decision maker's risk appetite. The process is demonstrated through a case study conducted in a global manufacturing supply chain involving semi-structured interviews and focus group sessions with experts in risk management. Theoretically grounded in the framework of Bayesian Belief Networks (BBNs) and Expected Utility Theory (EUT), the modelling approach has a number of distinctive characteristics. It utilises a top-down approach of Fault Tree Analysis (FTA). Performance measures are identified first and subsequently connected to risks. A 'probabilityconditional expected utility' matrix is introduced to reflect the propagation impact of interdependent risks on all performance measures identified. A 'weighted net evaluation of risk mitigation' method is proposed and the method of 'swing weights' is used to capture the trade-off between the efficacy of strategies and the associated cost keeping in view the decision maker's risk appetite. The approach adapts and integrates techniques from safety and reliability engineering (FTA), decision making under uncertainty (EUT), and multi-criteria decision analysis (swing weights). The merits and challenges associated with the implementation of interdependency based frameworks are discussed. Propositions are presented to elucidate the significance of modelling interdependency between risks and strategies. Keywords: Supply Chain Risk Network Management, Interdependencies, Multiple Performance Measures, Risk Mitigation Strategies, Bayesian Belief Networks, Expected Utility.
\end{abstract}

\section{Introduction}

Aiming for competitive advantage, firms operating across the global marketplace are exposed to considerable risk (Tang \& Tomlin, 2008; Christopher et al., 2011, Blos \& Miyagi, 2015). The commencement of research in supply chain risk management (SCRM) dates back to the early years of 21st century (Harland et al., 2003, Christopher \& Peck, 2004, Manuj \& Mentzer, 2008). There is an extensive literature on SCRM that considers conceptual theory building facets as well as empirical investigations of best practice in managing risks. This literature has been well-considered in numerous literature reviews (Jüttner et al., 2003, Tang, 2006, Tang \& Nurmaya Musa, 2011; Colicchia \& Strozzi, 2012; Heckmann et al., 2015). There are two major research gaps that necessitate immediate attention: first, the existing SCRM processes/frameworks have limited focus on the interdependency modelling 
of risks (Colicchia \& Strozzi, 2012, Garvey et al., 2015, Ho et al., 2015, Qazi et al., 2017); and second,

the risk appetite of a decision maker is not exclusively captured in general while prioritising risks and risk mitigation strategies (Heckmann et al., 2015).

Supply chains operate within an integrated setting of interdependent firms and even within a single firm, entities and risks are not isolated; rather, there are complex chains of interaction. Classification of supply chain risks has been explored comprehensively resulting in identification of independent categories of risks for aiding the risk identification stage of the SCRM process (Manuj \& Mentzer. 2008; Ho et al., 2015; Heckmann et al. 2015). However, risk identification must involve different stakeholders and capture the interdependent interaction between risks ranging across the entire supply network (Ackermann et al. 2014). Current risk classification schemes and methods investigating optimal treatment of individual risks can prove to be sub-optimal if there are correlations between risks and strategies (Garvey et al., 2015). According to Ho et al. (2015): "Investigating the joint impact of such risks can lead to better management of supply chains than treating each risk type in isolation. ... However, there is lack of research measuring the correlations between risk factors and corresponding risk types, or the probability of occurrence of particular risk types associated with their factors" (Ho et al. 2015, p. 5060).

The risk appetite of a decision maker drives the tolerance level with respect to the acceptance of risks and therefore, it is extremely important to integrate risk appetite within the decision making framework. According to Heckmann et al. (2015, p. 127): “The decision maker's degree of acceptance with respect to the deterioration of target-values defines his attitude towards supply chain risk. Riskaverse supply chain managers only accept a minor deterioration of target values of an efficiency- (or effectiveness-) based supply chain goal in exchange for the adherence or increase of an effectiveness(or efficiency-) based supply chain goal. Risk-seeking decision makers, however, accept higher degrees of value deterioration of a specific goal in exchange for the adherence or increase of an opposite one. Risk-neutral supply chain managers prefer neither of the two objective types". Very few frameworks in SCRM have captured the risk appetite of a decision maker, and where they have risks are treated as independent (Knemeyer et al. 2009; Lavastre et al., 2012). To the best of the authors' knowledge, no existing study has ever investigated designing a risk management framework within a network setting of interacting risks in which the risk appetite of a decision maker is taken into account.

Risk mitigation strategies are implemented in order to reduce the likelihood of occurrence and/or negative impact of risks (Tang \& Tomlin, 2008). Robust strategies must be developed in order to help firms reduce cost and/or improve customer satisfaction under normal conditions and enable firms to sustain operations during and after the occurrence of a disruption (Tang, 2006). Despite the significance of evaluating risk mitigation strategies specific to a portfolio of supply chain risks, very few studies have proposed tools to prioritise strategies subject to a budget constraint (Micheli et al., 2014; Aqlan 
\& Lam, 2015; Qazi et al., 2017), and where they have risk preferences have not been taken into

account. Although Expected Utility Theory (EUT) provides a standardised normative framework to make decisions under uncertainty, it is not so much used in practice mainly because of the difficulty associated with assigning utility values to all possible outcomes (Aven \& Kristensen, 2005).

A number of established techniques have been utilised to identify, assess and treat supply chain risks (Colicchia \& Strozzi, 2012; Ho et al., 2015). Fault Tree Analysis (FTA) being widely used in the literature on system safety and reliability engineering (Ashrafi et al., 2015) is considered an effective technique for managing supply chain risks (Oehmen et al., 2009; Sherwin et al., 2016). The technique is used to identify a top event (occurrence of a risk) and develop a network of causal factors leading to the top event. It can serve as a useful framework for identifying performance measures (top events) and developing a risk network (comprising supply chain risks) leading to the performance measures. However, there is a need to overcome the limitation of FTA by means of modelling common cause factors within the risk network. Bayesian Belief Networks (BBNs) provide a very useful framework for capturing probabilistic interdependency between uncertain variables and some recent studies have proposed BBN based frameworks to model and assess supply chain risks (Garvey et al., 2015, Qazi et al., 2017).

There are several contributions of this paper. First, we develop and operationalise a supply chain risk network management (SCRNM) process that captures interdependencies between risks, multiple (potentially conflicting) performance measures and risk mitigation strategies helping to prioritise risks and strategies specific to the decision maker's risk appetite, and demonstrate its application through a case study. Second, the proposed operationalisation scheme adapts established techniques from safety and reliability engineering, decision making under uncertainty and multi-criteria decision analysis, and integrates these together across different stages of the risk management process: risk identification- BBNs and FTA are utilised to develop a risk and performance network thereby capturing interdependency between risks (including common cause failures) and focussing exclusively on material risks specific to the performance measures identified; risk analysis- BBNs and EUT are used to assess and map risks on the proposed 'probability-conditional expected utility' matrix in order to capture the impact of risks on all performance measures rather than a single monetary measure used in the conventional risk matrix based tools; risk treatment- 'weighted net evaluation of risk mitigation' is introduced and the method of 'swing weights' (Belton \& Stewart, 2002) utilised to establish the trade-off between efficacy of potential risk mitigation strategies and the associated cost considering the decision maker's risk appetite. There is an added benefit of the process as besides modelling interdependency between risks, the utility for both risk appetite and trade-off across performance measures is exclusively captured whereas modelling these features in silo would undermine the integrated effect of complex interactions involved. Third, we present merits and challenges associated with the imple- 


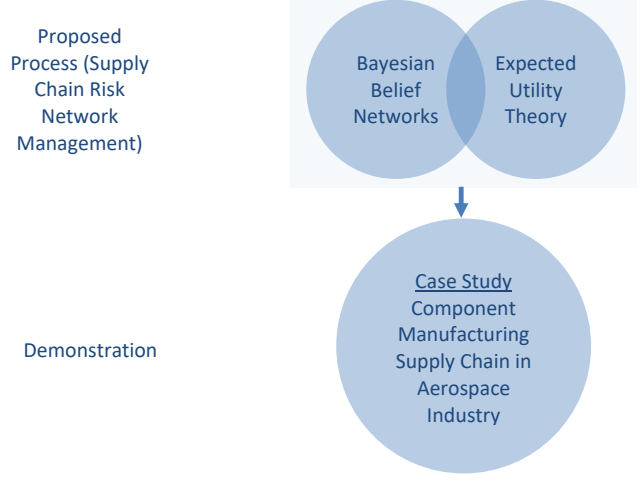

Figure 1: Research focus and methodology.

mentation of such interdependency based frameworks. Fourth, we develop propositions to elucidate the importance of accounting for interdependence of risks and risk mitigation strategies.

Responding to the call for developing and empirically evaluating a SCRM process that not only captures interdependency between risks but also integrates all stages of the process (Colicchia \& Strozzi, 2012; Ho et al., 2015), we address the following research questions in this study:

RQ1: How can we develop and operationalise a SCRM process that captures interdependencies between risks, multiple (potentially conflicting) objectives (performance measures) and risk mitigation strategies specific to the risk appetite of a decision maker?

RQ2: What are the merits and challenges associated with the implementation of the proposed process?

An overview of the research focus and the methodology adopted is shown in Figure1. The remainder of the paper is organised as follows: An overview of the relevant literature is presented in Section 2 , The proposed process and the methodology are described in Sections 3 and 4 , respectively. The case study conducted for demonstrating the application of the proposed process is presented in Section 5 . We discuss the implications of our findings and introduce propositions in Section 6. Finally, we present conclusions and directions for future research in Section 7 . 


\section{Literature Review}

In the following sections, we give an overview of the literature on supply chain risk management process and interdependency modelling of supply chain risks.

\subsection{Supply Chain Risk Management Process/Framework}

SCRM is "the identification and management of risks for the supply chain, through a co-ordinated approach amongst supply chain members, to reduce supply chain vulnerability as a whole" (Jüttner et al., 2003, p. 201). Several risk management frameworks have been proposed using different terminology; however, there is a consensus that a SCRM process involves five sequential stages: risk identification; assessment; analysis; treatment; and monitoring (Giannakis \& Papadopoulos, 2016). Selected articles conforming to the research focus of the paper (see Figure 1) have been classified into four categories including interdependency modelling of risks, the risk appetite of a decision maker, interdependency between risks and strategies, and research methodology as shown in Table A.1 (see Appendix A.

Ritchie \& Brindley (2007) identified five components of a SCRM process: risk drivers (primary and secondary level); risk management influencers (rewards, supply chain risks, timescales, portfolio); decision maker characteristics (perceptions, risk profile, attitudes, experiences); risk management responses (risk taking, avoidance, mitigation, monitoring); and performance outcomes (profit related, strategic positioning, personal). We will briefly describe the merits of some of the frameworks proposed in the literature, and delineate the main limitations of these.

A number of qualitative frameworks have been proposed to identify risks and prescribe generalised strategies to deal with important risks. These frameworks generally utilise qualitative scales to discretise the conventional risk matrix across the probability and impact levels. Utilising a Failure Modes and Effects Analysis (FMEA) based technique, Sinha et al. (2004) developed a process to manage risks in the aerospace industry whereas Giannakis \& Papadopoulos (2016) proposed a risk management process to identify and manage sustainability related risks across the environmental, social and economic facets with its application demonstrated through empirical case studies and a survey questionnaire. Khan et al. (2008) reported the conventional risk matrix based process used in a major UK retailer that helps the company deal with design oriented supply chain risks. Bringing the perspective of a global supply chain and consolidating the concepts from logistics, supply chain management, operations management, strategy and international business management, Manuj \& Mentzer (2008) proposed a procedure to help global supply chain managers identify risks and select appropriate strategies.

Quantitative frameworks have utilised hybrid methods to assess and manage risks. For example, Elleuch et al. (2014) combined FMEA, design of experiments, discrete event simulation, Analytical Hierarchy Process (AHP) and desirability function approach to develop a process and applied it to a 
pharmaceutical supply chain case study. Similarly, Aqlan \& Lam (2015) proposed a hybrid approach

of bow-tie analysis and stochastic integer programming to identify critical risks and assess suitable strategies taking into account their cost and effectiveness in reducing the risk exposure. Systems thinking has also been applied to develop a comprehensive process both in its qualitative (Oehmen et al. 2009) and quantitative forms (Ghadge et al., 2013).

There are mainly three limitations of the existing frameworks including the aforementioned studies. First, the frameworks have drawn limited focus on modelling the common cause failures and assessing their propagation impact. As such common cause failures can have a far reaching impact on the efficiency of a supply network, there is a need to model and evaluate such factors (Ho et al., 2015). Second, researchers generally focus on limited stages of the risk management process whereas "there is a significant relationship between all SCRM processes, (therefore) more attention should be given to legitimately integrated processes instead of individual or fragmented processes. ... Similarly, the effectiveness of risk mitigation strategies requires explicit quantification of effectiveness and efficiency of such strategies" (Ho et al., 2015, p. 5053). Third, there has been a very limited focus on the need for integrating risk appetite in the risk management process as Heckmann et al. (2015) argue that "More advanced (context-sensitive) approaches especially with respect to the risk attitude of the decision maker and with respect to the environment of the affected supply chain are needed" (Heckmann et al., 2015, p. 130). We endeavour to fill the mentioned gaps by developing and empirically evaluating an integrated SCRNM process to establish how practitioners perceive correlations between risks specific to their risk appetite and whether they are able to evaluate the impact of risk mitigation strategies on the network of risks.

\subsection{Interdependency Modelling of Supply Chain Risks}

Various models have been proposed to capture interdependency between supply chain risks. Interpretive structural modelling (ISM) is a hierarchy based technique that establishes the order and direction of complex relationships among elements of a system. It has been used to determine causal relationships between risk mitigation strategies (Faisal et al. 2006) and supply chain risks (Pfohl et al. 2011). Related to the same family of causal mapping techniques, fishbone diagram has been utilised to identify cause-effect relationships between risks (Lin \& Zhou, 2011). Mapping a supply network as a web of interconnected nodes, measures from the Social Network Analysis have been adapted to identify critical supply nodes (Kim et al. 2011$)$. The main limitation of aforementioned techniques is the inability to capture the strength of interdependency between risks.

AHP is a technique to conduct pair-wise comparisons between variables and identify their relative importance. Its application varies from the risk assessment of suppliers (Ganguly, 2014) to the prioritisation of supply chain performance measures (Gaudenzi \& Borghesi, 2006). FMEA is a technique to prioritise risks depending on the relative product of probability, severity and detectability associ- 
ated with each risk. It has been extensively used in SCRM to identify critical risks (Nepal \& Yadav, 2015; Dong \& Cooper, 2016). Similarly, utilising established techniques from the field of reliability engineering, Aqlan \& Lam (2015) proposed a bow-tie analysis based process to capture the interdependency of supply chain risks whereas Oehmen et al. (2009) and Sherwin et al. (2016) introduced FTA based frameworks to assess risks. The main problem with these techniques is their limited focus on capturing common cause failures. Although the conventional FTA does not capture common cause failures, the technique following a top-down approach is helpful in terms of brainstorming the causes of a consequence and is widely used in the literature on engineering risk management (Sherwin et al. 2016).

Mainly, supply chain risks are classified into distinct categories like process, control, demand, supply and environmental risks (Christopher \& Peck, 2004). The first two risk categories relate to factors internal to an organisation, the third and fourth include factors internal to the supply chain, but external to the organisation and the fifth category relates to factors external to the supply chain. Similar to the concept of mapping causal chains in project risk management (Ackermann et al., 2014), Badurdeen et al. (2014) proposed a risk taxonomy capturing interdependency between supply chain risks that is in contrast with the established classification schemes.

In response to the call for understanding the relationships between a set of strategies for managing risks and corresponding impact on performance measures (Colicchia \& Strozzi, 2012), a few models have been developed (Micheli et al., 2014; Aqlan \& Lam, 2015); however, these models do not explicitly capture interdependency between risks. Another issue relates to the focus of these models on "minimising cost or maximising profit as a single objective" (Colicchia \& Strozzi, 2012, p. 412) as "purely cost-and waste-considering objectives, however, evaluate supply chain's performance in retrospect. They miss to assess both operational effectiveness and important strategic achievements like product quality and customer satisfaction" (Heckmann et al., 2015, p. 130). In this study, we overcome the limitation of earlier studies by not only capturing interdependencies between risks but also across the entire risk management process. We also consider optimising a set of potentially conflicting performance measures within an interdependent setting of interacting risks and strategies, and propose a new technique for prioritising risk mitigation strategies subject to a budget constraint.

\subsubsection{Application of Bayesian Belief Networks in Supply Chain Risk Management}

BBNs offer a unique feature of modelling risks combining both the statistical data and subjective judgement in case of non-availability of data (Sigurdsson et al., 2001; Kelangath et al., 2011). Although BBNs have been extensively used in the field of risk management (Norrington et al., 2008), their application to the field of SCRM is mainly focussed on addressing specific problems involving supplier selection, supplier assessment and ranking of suppliers. Recently, few models have been proposed to capture the supply network-wide web of risks. However, existing approaches have not considered 


\begin{tabular}{|c|c|c|c|c|c|c|c|c|c|}
\hline \multirow[b]{2}{*}{ Attributes } & \multirow[b]{2}{*}{$\begin{array}{c}\text { Lockamy and } \\
\text { McCormack } \\
(2009)\end{array}$} & \multicolumn{8}{|c|}{ Article } \\
\hline & & $\begin{array}{c}\text { Dogan } \\
\text { and Aydin } \\
(2011)\end{array}$ & $\begin{array}{c}\text { Leerojanap } \\
\text { rapa et al. } \\
(2013)\end{array}$ & $\begin{array}{l}\text { Badurdeen } \\
\text { et al. } \\
(2014) \\
\end{array}$ & $\begin{array}{l}\text { Garvey et } \\
\text { al. (2015) }\end{array}$ & $\begin{array}{l}\text { Nepal and } \\
\text { Yadav } \\
(2015)\end{array}$ & $\begin{array}{c}\text { Hosseini } \\
\text { and Barker } \\
(2016)\end{array}$ & $\begin{array}{l}\text { Qazi et } \\
\text { al. } \\
\text { (2017) }\end{array}$ & $\begin{array}{l}\text { This } \\
\text { paper }\end{array}$ \\
\hline $\begin{array}{l}\text { Risk } \\
\text { identification }\end{array}$ & $\mathrm{x}$ & $x$ & $\mathrm{x}$ & $x$ & $\mathrm{x}$ & $x$ & $x$ & $\mathrm{x}$ & $x$ \\
\hline $\begin{array}{l}\text { Risk analysis/ } \\
\text { evaluation }\end{array}$ & $x$ & $\mathrm{x}$ & $\mathrm{x}$ & $x$ & $x$ & $x$ & $\mathrm{x}$ & $x$ & $x$ \\
\hline Risk treatment & & & & & & & & $x$ & $x$ \\
\hline Risk appetite & & & & & & & & & $x$ \\
\hline $\begin{array}{l}\text { Multiple SC } \\
\text { performance } \\
\text { measures }\end{array}$ & & & & & & & & & $x$ \\
\hline $\begin{array}{l}\text { Modelling of } \\
\text { supply network } \\
\text { risks }\end{array}$ & & & $x$ & & $x$ & & & $x$ & $\mathrm{x}$ \\
\hline $\begin{array}{l}\text { New risk } \\
\text { measures }\end{array}$ & & & & & $x$ & & & $x$ & $x$ \\
\hline Real Case Study & $x$ & $x$ & $x$ & $x$ & & & & & $x$ \\
\hline Techniques & BBNs & BBNs & BBNs & BBNs & BBNs & $\begin{array}{l}\text { FMEA and } \\
\text { BBNs }\end{array}$ & BBNs & $\begin{array}{l}\text { FMEA, } \\
\text { BBNs, } \\
\text { Game } \\
\text { Theory }\end{array}$ & $\begin{array}{c}\text { FTA, } \\
\text { BBNs, } \\
\text { EUT, } \\
\text { MCDA }\end{array}$ \\
\hline
\end{tabular}

Table 1: Comparison of the Proposed Approach with Existing BBN Based SCRM Models.

framing and operationalising a comprehensive risk management process integrating suitable techniques across all stages of the process. Furthermore, the merits and challenges involved in implementing such a framework remain unexplored. A comparison of the merits of this paper with existing studies is presented in Table 1 .

Lockamy \& McCormack (2009) developed a model for benchmarking supplier risks involving risk events related to supplier network, internal operations and external factors. They used surveys and interviews for collection of data from both the internal and external company sources and applied the model on a group of 15 automotive casting suppliers for a major automotive company in US. Similarly, Badurdeen et al. (2014) proposed a tool for assessing supply risks and conducted sensitivity analysis to help Boeing company benchmark its Tier 1 suppliers. Addressing the supplier selection problem, Dogan \& Aydin (2011) developed a model combining Total Cost of Ownership and BBN methods and applied it in automotive industry to help Tier 1 suppliers select their own suppliers whereas Hosseini \& Barker (2016) introduced a framework focussing on resilience-based supplier criteria. The main problem with the utility of these models relates to their exclusive focus on a specific problem without adapting BBNs to the realm of SCRM and capturing complex nature of interdependent supply chain risks. For example, the criteria related to the supplier selection or benchmarking process are well established and BBNs can readily be applied to formulate a network structure without any adaptation. However, in the case of modelling interdependent risks across a supply chain, there is an added complexity involved in 
establishing the network structure that necessitates adapting BBNs to the context of SCRM whereas the literature does not provide an illustration of developing such models.

Leerojanaprapa et al. (2013) and Leerojanaprapa (2014) proposed a generic BBN modelling process to support supply chain risk analysis based on expert knowledge and conducted a case study in the medical supply chain to demonstrate its efficacy. In their effort to capture the probabilistic interdependency between supply chain risks, Garvey et al. (2015) introduced an algorithm to map risks and proposed supply chain risk measures. The main limitation of these studies is their focus on limited stages of the risk management process and ignoring the risk appetite of a decision maker. Also, modelling of risks in accordance with the process flow of a supply chain makes it infeasible to capture risks relating to substantial supply networks. However, these studies serve to illustrate the efficacy of BBNs in modelling and managing supply chain risks as BBNs can effectively measure the propagation impact of these risks within a network setting. Utilising BBNs, Qazi et al. (2017) introduced probabilistic supply chain risk measures to prioritise interdependent risks and strategies. Although one of the measures introduced captures an aversion to risk, the entire risk management process does not explicitly model the risk attitude (utility) of a decision maker and also, the process only helps in optimising a portfolio of strategies specific to a single performance measure (objective) rather than considering multiple (potentially conflicting) measures (objectives). We endeavour to fill these gaps through introducing and operationalising a comprehensive SCRNM process that adapts key features of established techniques from safety and reliability engineering, decision making under uncertainty and multi-criteria decision analysis to the context of SCRM.

\section{Proposed SCRNM Process}

There are many studies in the literature with exclusive focus on the impact of supply chain risks on performance measures (Jüttner et al., 2003; Zhao et al., 2013), however, the main limitation of these studies is modelling risks in silo whereas we focus on modelling a risk network and evaluating its holistic impact on performance measures. We also capture the moderating effect of a risk management process through modelling risk mitigation strategies within the risk network. Therefore, the established framework proposed by Jüttner et al. (2003) is modified to account for the holistic (negative) impact of a supply chain risk network on (potentially conflicting) supply chain performance measures as shown in Figure 2, Also, the efficacy of a risk management process influences the impact of a risk network on performance measures as selecting effective strategies would mitigate the consequence of risks. The steps involved in the risk management process are shown in Figure 3. Like the standard risk management process (SA, 2009), the proposed process starts with establishing the context in terms of defining the scope of the supply chain and its boundaries. The main purpose of the modelling and analysis is ascertained through interviewing the decision maker or the main stakeholders. The decision 


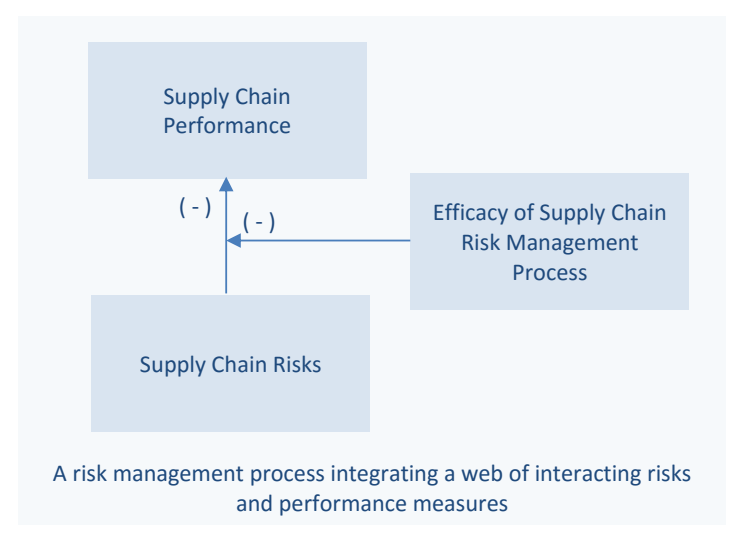

Figure 2: Supply chain risk management framework (adapted from Jüttner et al. 2003).

maker also helps in identifying the key performance measures pertinent to the supply chain.

A focus group session must be conducted to identify risks and develop a causal network. We found it very useful to develop the causal network using the top-down approach where the informants were asked to link each performance measure with the corresponding risk(s) that were in turn linked back to causal factors. In a way, it mimics the technique adopted in conventional FTA (Sherwin et al. 2016); however, FTA does not capture the common-cause failures whereas we model such factors in our framework. Studies on developing the qualitative part of the BBNs and causal maps are useful in establishing the risk network (Nadkarni \& Shenoy, 2004). Once the qualitative network is developed, there is a need to validate the structure and ensure whether all relevant risks have been considered. A focus group session involving all participants from the previous session and adding some new members is helpful in refining the structure and adding some missing risks. It is important to note that it is an iterative process until the final structure is validated and the participants are satisfied with the structure of the risk network.

The next stage relates to the quantitative modelling of the already validated qualitative risk network where the participants establish the strength of interdependency between the risks either through semistructured interviews or a focus group session. Once all the conditional probability values have been elicited, a focus group session must be held to validate the model. Again studies specific to the quantitative modelling of BBNs are useful in developing and validating the model (Norrington et al., 2008). Sensitivity analysis is carried out to evaluate the impact of individual risks on each performance measure and ascertain whether the results make sense and conform to the perception of the participants. In case of any discrepancy, the quantitative model is revisited and amendments incorporated until the sensitivity results are agreed upon.

Following the validation of a quantitative model, the decision maker is consulted with regard to the identification of potential risk mitigation strategies, associated cost and the budget constraint. A focus group session must be held to identify the connection of strategies with relevant risks and establish the efficacy of strategies in reducing the probability of risks. There is also a need for validating the efficacy 


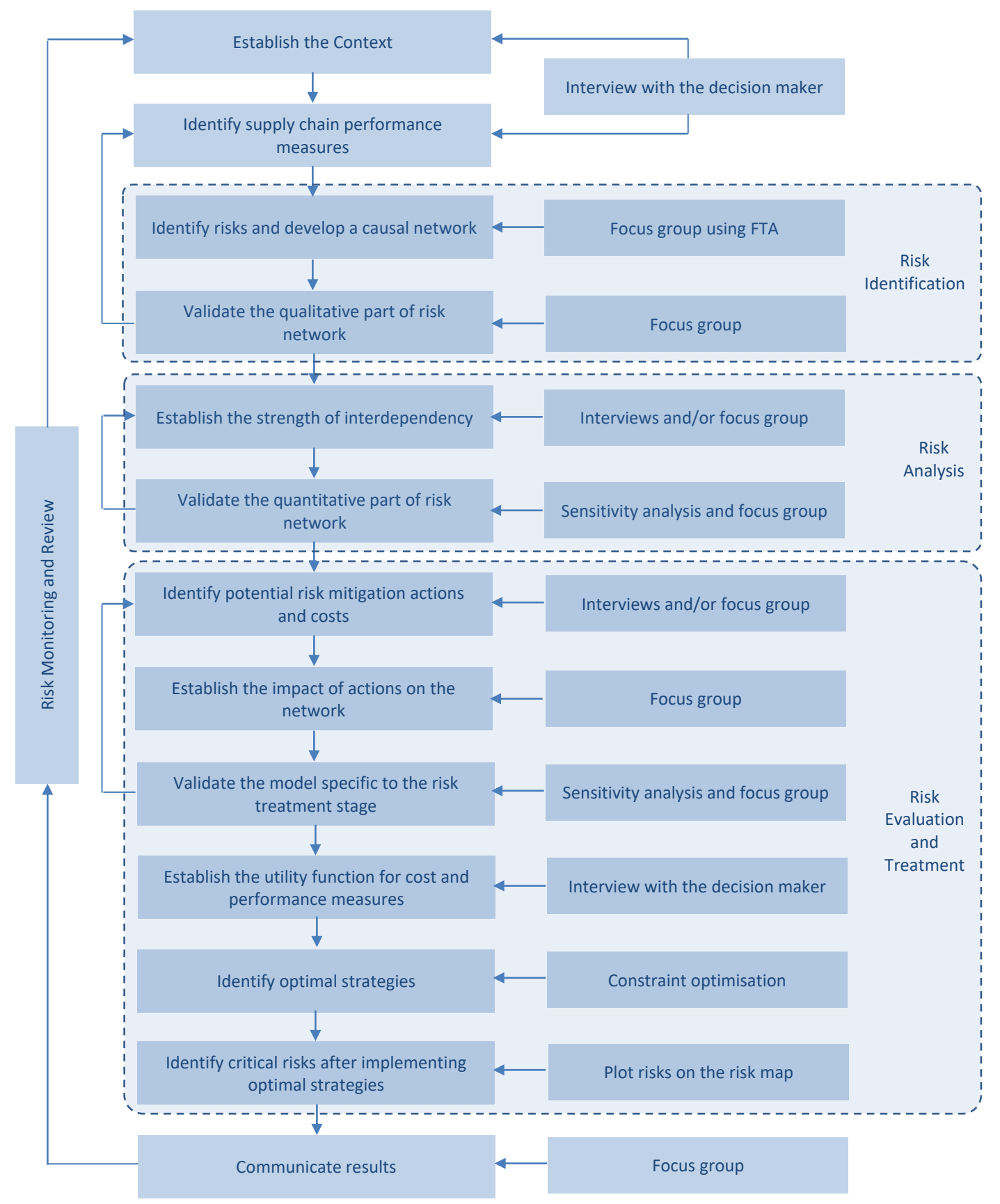

Figure 3: Proposed supply chain risk network management process. 
of risk mitigation strategies. The decision maker is again consulted to determine the utility function

corresponding to the performance measures and the cost of strategies. The model is subsequently run for all possible combinations of strategies subject to various budget constraints and the strategies are selected that maximise the overall expected utility of the decision maker. Finally, a focus group session is conducted to communicate the results to the participants and help the decision maker understand the impact of implementing different combinations of strategies. As risk management is a continuous process, the entire process is repeated requiring minimal changes in the model once new risks are discovered and updated.

\section{Methodology}

An important aim of this study is to empirically evaluate the proposed process through a case study in order to demonstrate the application of the process and establish the benefits and challenges associated with its implementation. The empirical evaluation of the process involved establishing the context of a specific organisation (case) and developing a model based on how the decision makers perceived interdependencies between risks and why certain risks and performance measures were given due importance (study). As the "case study method is an appropriate choice for investigating 'how' and 'why' questions" (Yin, 2009, p. 27), we adopted the same methodology to address the research questions.

Aero (a leading global technology provider) was selected for conducting the case study as their risk managers were keen on improving the risk management process within the company and assessing the merits and challenges of our proposed process. The initial interview protocol was piloted with Zanardi Fonderie (an Italian global manufacturing company specialising in the heat treatment of iron and its alloys) that helped in revising the questions to clarify the terms and adopting a well-structured method to develop the risk network in the case study. The main data collection method was semistructured interviews as "the overwhelming strength of the face-to-face interview is the 'richness' of the communication that is possible" (Gillham, 2000, p. 62) and "the semi-structured interview is the most important form of interviewing in case study research and it can be the richest single source of data" (Gillham, 2000, p. 63). As our research involved developing a risk network, the case study design utilised a mix of quantitative and qualitative evidence.

In order to obviate the chance of misrepresentation and loss of data, all interviews were audiotaped with the permission of respondents. Also, two researchers were engaged in conducting the case study in order to ensure the validity of research and the guidelines provided by Nadkarni \& Shenoy (2004) and Pitchforth \& Mengersen (2013) were strictly followed to validate the models developed. Following the interviews, the authors transcribed the recordings and validated the data internally and performed content analysis for data reduction and concept identification. Subsequently, the transcripts 
and deduced themes were shared with the interviewees for validation. Besides interviews, secondary

data including publicly available corporate reports, case studies and annual performance reports were collected and analysed in order to triangulate the data collected through interviews and focus group sessions. Finally, a case study report was prepared and shared with the company to validate the authenticity of results and help the participants identify any issues.

\subsection{Proposed Modelling Approach}

A number of techniques including but not limited to AHP, Analytical network process, Fuzzy set theory, ISM, Network theory, FMEA and hybrid methods integrating these have been extensively used in modelling supply chain risks. The main limitation of these techniques is the inability to comprehensively capture probabilistic interdependency between risks and to propagate and update beliefs upon receiving new information. Based on the efficacy of BBNs in capturing interdependencies between risks, we consider BBN based modelling of a risk network as an effective approach. Such a modelling technique can help managers visualise supply chain risks and take effective mitigation strategies. BBNs have already been explored in the literature on risk management (Ashrafi et al. 2015; Wu et al., 2015) and SCRM (Garvey et al., 2015; Nepal \& Yadav, 2015, Qazi et al., 2017) for modelling and assessing risks. However, to the best of the authors' knowledge, BBNs have not been explored in the literature on SCRM to establish the impact of supply chain risks on multiple (potentially conflicting) objectives and to prioritise supply chain risk mitigation strategies considering the risk appetite of a decision maker. Another contribution of the proposed approach relates to the adaptation of established techniques from safety and reliability engineering, decision making under uncertainty and multi-criteria decision analysis, and integrating these together to operationalise the proposed process.

Interdependency modelling has been extensively explored in other research areas especially the reliability and safety of engineering systems and also, well-established techniques like BBNs and EUT are commonly used in capturing interdependency between risks and modelling the risk appetite of a decision maker, respectively (Aven \& Kristensen, 2005, Aven, 2015). However, these methods and risk management frameworks are not readily (directly) applicable to modelling and managing supply chain risks mainly because of the complex and unique features of supply chain risks: unlike risks associated with engineering (physical) systems, supply chain risks involve soft factors like people risks and corporate governance issues; the layout (qualitative causal structure) of a physical system is generally known whereas it is very difficult (not viable) to accurately model a supply network and corresponding risks because of the number of suppliers and entities involved; components within a physical system can readily be monitored for any malfunction whereas it might not be possible to detect a risk occurring within a supply chain where not all stakeholders are incentivised to share any private information with regards to the realisation of a risk or their reliability; engineering systems are maintained and improved 
through the use of established interdependency based models and maintenance (and accident) data

recorded whereas such data is not readily available in the case of supply chain risks as practitioners rely on risk matrix based tools and interdependency modelling is generally ignored (Leerojanaprapa, 2014). Therefore, there is a need to adapt the interdependency based tools commonly used in other areas to the context of SCRM such that the complexity associated with supply chain risks is managed effectively and the tools developed fit well with the requirements and competence of practitioners who prefer to use simple risk matrix based tools.

In order to capture the risk appetite of a decision maker, we make use of EUT. However, instead of utilising the conventional technique to elicit a decision maker's preference over the entire combination of risks, we introduce a new approach of mapping the combination of risks to a set of performance measures making it feasible for capturing the risk appetite over a substantial risk network. This adaptation not only reduces the elicitation burden of ascertaining utility values but also helps in evaluating risks specific to global supply chains where the complex nature of interdependency between risks is not amenable to conventional SCRM techniques. Integrating BBNs with EUT provides an added benefit as besides modelling interdependency between risks, the utility for both risk appetite and trade-off across performance measures is exclusively captured whereas modelling these features in silo would undermine the integrated effect of the complex interactions involved. As such, the complexity relates to the interdependent nature of supply chain risks, non-linear interactions between risks and performance measures, utility of the decision maker with regards to the trade-off across these measures, and their risk appetite in terms of establishing the maximum level of risk exposure and the utility of risk mitigation with regards to the cost incurred in introducing strategies. Our approach deals with capturing this complexity in a unique manner and integrates all these features in a holistic framework. In the following sections, we provide a brief overview of the BBNs and EUT as these have been used to develop the proposed modelling approach.

\subsubsection{Bayesian Belief Networks}

$\mathrm{BBN}$ is a graphical framework for modelling uncertainty. BBNs have their background in statistics and artificial intelligence and were first introduced in the 1980s for dealing with uncertainty in knowledge-based systems (Sigurdsson et al. 2001). They have been successfully used in addressing problems related to a number of diverse specialties including reliability modelling, medical diagnosis, geographical information systems, and aviation safety management among others. For understanding the mechanics and modelling of BBNs, interested readers may consult Sigurdsson et al. (2001); Nadkarni \& Shenoy (2004); Jensen \& Nielsen (2007). A BBN consists of the following elements:

- A set of variables (each having a finite set of mutually exclusive events) and a set of directed edges between variables forming an acyclic directed graph; a directed graph is acyclic if there is 
no directed path $X_{1} \rightarrow \ldots \rightarrow X_{n}$ so that $X_{1}=X_{n}$, furthermore, the directed edges represent sta-

tistical relations if the $\mathrm{BBN}$ is constructed from the data whereas they represent causal relations if they have been gathered from experts' opinion,

- A conditional probability table $P\left(X \mid Y_{1}, \ldots Y_{n}\right)$ attached to each variable $X$ with parents $Y_{1}, \ldots, Y_{n}$.

Chain Rule for Bayesian Belief Networks. Let a Bayesian Network be specified over $X=X_{1}, \ldots, X_{n}$. The structure of a BBN implies that the value of a particular node is conditional only on the values of its parent nodes. Therefore, the unique joint probability distribution $P(X)$ representing the product of all conditional probability tables is given as follows:

$$
P(X)=\prod_{i=1}^{n} P\left(X_{i} \mid p a\left(X_{i}\right)\right)
$$

where $p a\left(X_{i}\right)$ are the parents of $X_{i}$.

Merits and Challenges. BBNs present a useful technique for capturing interdependency between supply chain risks (Badurdeen et al. 2014). Another advantage of using BBNs for modelling supply chain risks is their ability of back propagation that helps in determining the probability of an event that may not be observed directly. They provide a clear graphical structure that most people find intuitive to understand. Besides, it becomes possible to conduct flexible inference based on partial observations, which allows for reasoning. Another important feature of using BBNs is to conduct what-if scenarios. There are certain problems associated with the use of BBNs: along with the increase in number of nodes representing uncertain variables, a considerable amount of data is required in populating the network with (conditional) probability values; similarly, there are also computational challenges associated with the increase in number of nodes.

\subsubsection{Expected Utility and Decision Making under Uncertainty}

Within the context of decision making under uncertainty, risk can be related to a utility function that reflects the preferences of a decision maker with regard to various possible consequences of a decision. Expected utility theory posits that a decision-maker's preferences over an outcome $x$ can be represented by a utility function $u(x)$, and if there are $i=1, \ldots, n$ possible states of the world each of which occurs with probability $p_{i}$ and in which the outcome is $x_{i}$ then the decision-maker cares about their expected utility $\sum_{i=1}^{n} p_{i} u\left(x_{i}\right)$. Faced with a set of alternatives, a decision maker will choose among those alternatives by selecting the option that yields the highest expected utility. The shape of the utility function captures the risk attitude of a decision maker: for a risk averse individual it will be concave; for risk seeking convex; and for a risk neutral individual it will be linear. Risk averse (seeking) decision makers would always choose (decline) a certain option over a risky option with the same expected value; so in particular risk averse individuals need compensating for taking on risk. 


\subsubsection{Proposed Approach}

Although EUT provides a standardised normative framework to make decisions under uncertainty, it is not so much used in practice mainly because of the difficulty associated with assigning utility values to all possible outcomes (Aven \& Kristensen, 2005). If a network consists of $N$ risks each of which has binary outcomes then there are $2^{N}$ utility values that must be elicited, which is potentially a very large number. To circumvent this difficulty, we introduce a new approach to evaluating a network of interconnected risks.

Let a risk network be combined of $j=1, \ldots, N$ interdependent binary risks denoted $R_{j}$ that can take the value 'true' or 'false'. Rather than assessing the state of each risk, it will be assumed that the combination of these risks can be summarised in $M<N$ binary performance measures denoted $m_{l}$, $l=1, \ldots, M$, that can take the value 'good' or 'bad'. The probability that each risk is realised in the network combines to determine the probability of each performance measure being 'good' or 'bad'.

A state of the network is a particular realisation of performance measures, each of which can be either 'good' or 'bad'. We denote a typical state $s_{i} \in\{\operatorname{good}, b a d\}^{M}$; there are $2^{M}$ possible states in the set of states that we denote by $I$. Decision makers are assumed to evaluate a realisation of the network in a particular state by the combination of performance measures that are realised in that state. As such, we define the decision-maker's utility function as

$$
u:\{\text { good, bad }\}^{M} \rightarrow[0,1]
$$

where utility evaluations are scaled to take a value on the unit interval. The probability that state $i$ occurs is the joint probability that each of the performance measures takes its value specified by the state, that we denote $p_{i}$. Decision makers are then assumed to evaluate the expected utility of the network:

$$
E U=\sum_{i \in I} p_{i} u\left(s_{i}\right)
$$

As the state of risks influences performance measures, we introduce the notion of risk propagation measure $(R P M)$ to capture the relative impact of each risk on the set of performance measures modelled within a risk network. $R P M_{j}$ is the probability weighted expected utility of the network if risk $j$ is realised.

$$
R P M_{j}=p\left(R_{j}=\text { true }\right) E U \mid R_{j}=\text { true }
$$

We will now consider that the decision maker has a set of $a=1, \ldots, A$ risk mitigation actions available that change the probability of some risks occurring and therefore, because of interdependence between risks, influence the whole network of risks. These actions can be combined in any way to 
form a mitigation strategy and we denote a typical risk mitigation strategy by $\sigma_{k}$. We let $\sigma_{0}$ denote

the (costless) strategy 'do nothing' so the network is in its original configuration; $\sigma_{2^{A}-1}$ means do everything. There are $2^{A}$ combinations of risk mitigation actions (including doing nothing) in the set of mitigation strategies that we denote $K$. By undertaking a risk mitigation strategy the probability with which each state of the network (in terms of performance measures) is realised is influenced, and therefore we write these probabilities as a function of the risk mitigation strategy adopted, $p_{i}\left(\sigma_{k}\right)$. Risk mitigation is a costly exercise; we write $C_{k}$ as the cost of undertaking the strategy $\sigma_{k}$. To evaluate a realisation of the network after undertaking a mitigation strategy, we should write a decision-maker's utility as a function of both $s_{i}$, the state of the performance measures and $C_{k}$, the cost of mitigation, so the expected utility resulting from undertaking risk mitigation strategy $k$ would be

$$
\sum_{i \in I} p_{i}\left(\sigma_{k}\right) U\left(s_{i}, C_{k}\right)
$$

However, operationally this specification would require that we elicit utility values over each state of the network in every possible cost realisation, which is often not feasible. To circumvent this problem, we assume that utility is separable in the evaluation of the state of the network and the cost of mitigation (Wilson \& Quigley, 2016). To scale the evaluation of the cost of mitigation strategies we define a utility value for the cost $v\left(C_{k}\right)$ that yields a utility of 1 if there is no cost and then reduces to a minimum value of zero as the cost of mitigation increases. Further, we consider a 'weighted net evaluation' (WNE) of a mitigation strategy, which is defined as

$$
W N E\left(\sigma_{k}\right)=(1-\alpha) E U\left(\sigma_{k}\right)+\alpha v\left(C_{k}\right)
$$

where

$$
E U\left(\sigma_{k}\right)=\sum_{i \in I} p_{i}\left(\sigma_{k}\right) u\left(s_{i}\right)
$$

$\alpha$ captures the importance of cost to the decision maker, and we propose using the method of 'swing weights' (Belton \& Stewart, 2002) to determine the value of this parameter. The decision maker is first asked to consider that both utility (relative to objectives) and cost are at the least preferred states (all risks realised and maximum possible cost of strategies incurred amounting to the utility value of 0 ). Subsequently, he is given a scenario that only one of these could be improved to the best possible state (giving a utility of 1) and is asked which he would prefer; the one picked by him should receive the maximum weight (100) reflecting the significance of that criterion. He is then required to assess the overall value (over a scale of $0-100$ ) arising from a swing from 0 to 1 on the other criterion. Letting this be $x$, the weight assigned to the chosen criterion is $100 /(100+x)$ and that assigned to the other is $x /(100+x)$. This process thus determines the value of $\alpha$ for a decision maker. 
Problem of Selecting Optimal Risk Mitigation Strategies. Having defined the way in which decision

makers assess the outcome of undertaking a mitigation strategy, we now define the objective function of a decision maker, which is to choose the mitigation strategy that maximises the WNE of risk mitigation, subject to the constraint that the cost of mitigation must not exceed a threshold, $\bar{C}$ :

$$
\max _{\sigma_{k} \in K} W N E\left(\sigma_{k}\right) \text { s.t. } C_{k} \leq \bar{C} \text {. }
$$

\section{Application of the Proposed Process}

\subsection{Description of the Case Study}

Founded in the early 20th Century, Aero is a leading global supplier of products, solutions and services within rolling bearings, seals, mechatronics, services and lubrication systems. Having 120 manufacturing units established in 29 countries and a distribution network across 130 countries, Aero serves a diversified mix of industries, including cars and light trucks, marine, aerospace, renewable energy, railway, metal, machine tool, medical and food and beverage.

The respondents were selected on the basis of their expertise in risk management in general and $\mathrm{SCRM} /$ project risk management in particular. A total of seven semi-structured interviews were conducted with details of the experts given in Table A.2. Each interview lasted for 90 minutes on average (with the minimum and maximum time of 70 and 120 minutes, respectively). A total of three focus group sessions were also held involving the development and validation of the model and communication of the results with each session lasting for 2 hours on average.

\subsection{Model Development and Results}

Five performance objectives namely quality, timeliness, market share, profit and sustainability were identified during the interviews. These objectives are interrelated as market share influences the profit margin and also, quality, timeliness and profit are potentially conflicting objectives. Instead of following a bottom-up approach as adopted in the Event Tree analysis, we developed the network using the FTA that utilises a top-down approach. The network was developed involving two members from the risk management group. They were asked to focus on a one-year time horizon and assess the probability of risks within that timeframe. Furthermore, the main focus was on identifying only main risks that would ultimately influence the performance objectives of the company. This exercise of brainstorming and linking risks to the performance measures identified (as used in the FTA) was guided by the principles of modelling a BBN (Nadkarni \& Shenoy, 2004).

Once the qualitative structure of the network was developed, two other members of the group were involved in validating the structure. Some changes were suggested by the members in terms of adding new nodes to the network like financial issues and communication plan among others that were finally included after deliberation. The final qualitative part of the model is shown in Figure 4 with details of 
risks presented in Table A.3. One main feature of the developed structure is capturing interdependency

between risks ranging across different categories namely supply, demand, process and control risks (Christopher et al., 2011) and therefore, instead of conceptualising risks into distinct categories, we focus on intra- and inter- dependency across all such categories in the form of a risk network as shown in Figure 5. Control risks represent the problems associated with the management policies and these can be considered as the common causes affecting the entire network of risks as shown in Figure 4. For example, poor management policies might adversely affect the motivation of employees which in turn would influence the production rate and even the quality might be compromised triggering customer dissatisfaction.

Following the qualitative validation of the risk network, another focus group session was held to quantify the model. Two of the participants were engineers and well conversant with the fundamentals of probability theory and therefore, it was not difficult to elicit conditional probability values. However, not all participants were comfortable with providing the probability values. Therefore, a qualitative scale was introduced to elicit probability and utility values as shown in Figure A.1 and Figure A.2, respectively.

The quantitative part was validated through conducting the sensitivity analysis during which some conditional probability values had to be revised as the participants were not satisfied with some of the sensitivity results. The updated probabilities of the quality (low), timeliness (delayed), market share (low), profit (low) and sustainability (low) were calculated as 0.35, 0.08, 0.68, 0.60 and 0.33, respectively. The (red) shade of a node represents its relative importance for the utility node in terms of the propagation impact whereas the thickness of an arc reflects the strength of interdependency (influence) between the interconnected nodes. The participants agreed with the optimistic results for quality, timeliness and sustainability and somehow justified their concern with regard to the higher probabilities associated with market share and profit. The results also conformed to their perception about the efficacy of already implemented strategies.

The decision maker was interviewed to determine the 'utility' associated with different values of the objectives as shown in Table A.4 and potential mitigation strategies were identified during another focus group session with associated costs shown in Table A.5. The strategies were finally mapped on the risk network as shown in Figure A.3 and the impact of each strategy was established through eliciting the relevant conditional probability values. The rectangular shaped nodes (except the objectives appearing at the top) represent all possible strategies. Once all the potential strategies were implemented, the updated probabilities of the quality (low), timeliness (delayed), market share (low), profit (low) and sustainability (low) were calculated as $0.23,0.05,0.37,0.33$ and 0.24 , respectively. The efficacy of strategies elicited was validated through conducting sensitivity analysis.

The model was simulated for each possible combination of strategies $\left(2^{9}\right.$ iterations $)$ and the expected 


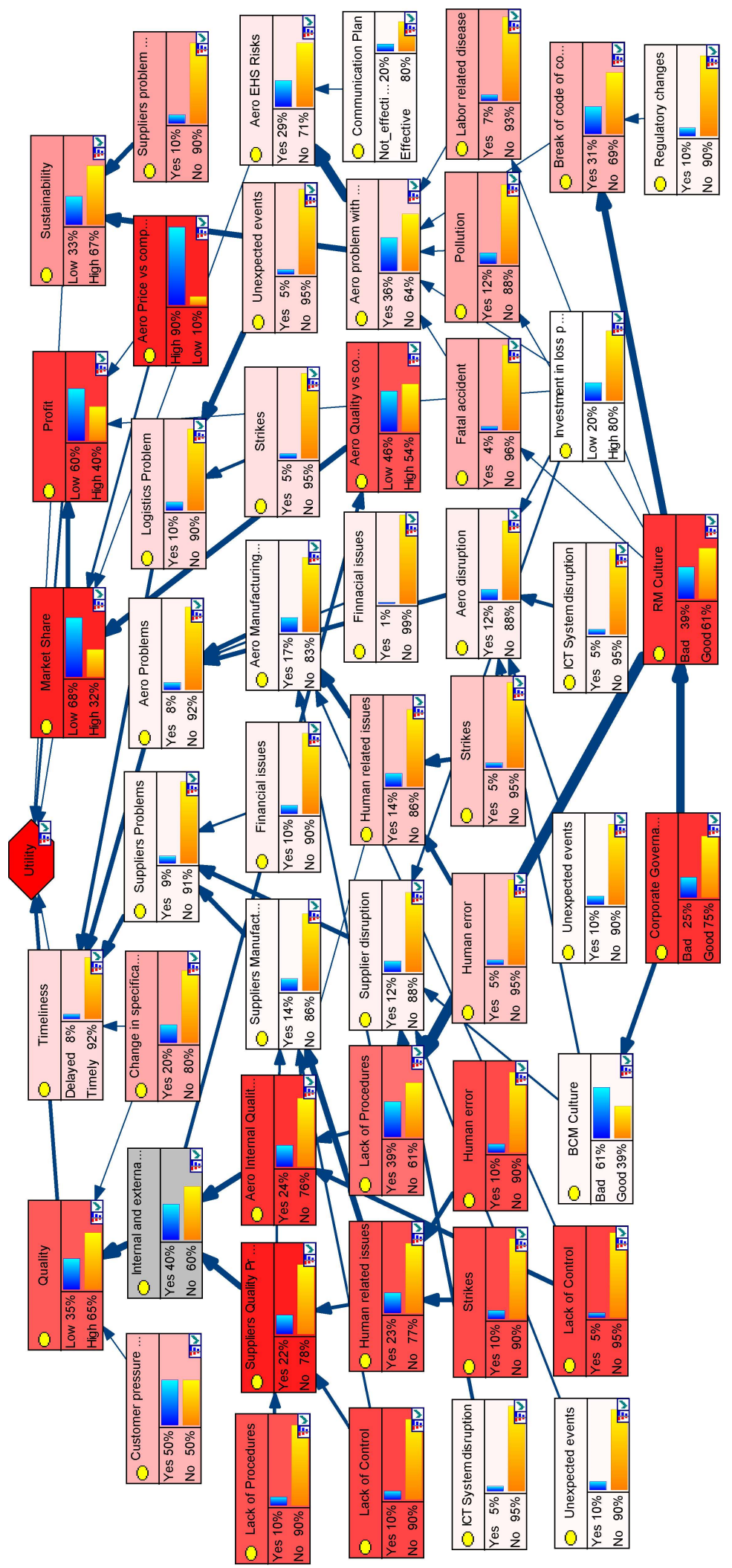

Figure 4: Network of interacting risks and risk sources with no potential strategies implemented (GeNIe). 
utility value evaluated for each instance. Figure 6 plots the cost and expected utility combinations for each of the strategies. If a decision maker was targetting a particular cost of implementation they should choose the strategy that gives the highest expected utility for that cost. Note that increasing the cost of mitigation does not always improve utility, as can be seen in the range $195-235$ units of cost.

The maximum weighted net evaluation of risk mitigation was mapped subject to different weights assigned to the expected utility and cost of strategies as shown in Figure 7. This graph provides as a validity check as the cost of the optimal solution is decreasing as the weight attached to cost increases (higher $\alpha$ ). Next, the optimal investment level (one maximising the $W N E$ of risk mitigation) with respect to the budget constraint was determined as shown in Figure 8. A decision maker assigning equal importance to the improvement in expected utility value and the mitigation cost must never invest in strategies costing more than 30 units. Similarly if the decision maker attributes $90 \%$ of the importance to the improvement in expected utility, the investment level should be increased to 165 units.

The efficacy of undertaking a risk mitigation strategy can be conveniently illustrated to decision makers. With the network in a particular state, consider a plot of the probability of each risk being realised against the expected utility consequence if it is realised, as in Figure 9. Following Ruan 


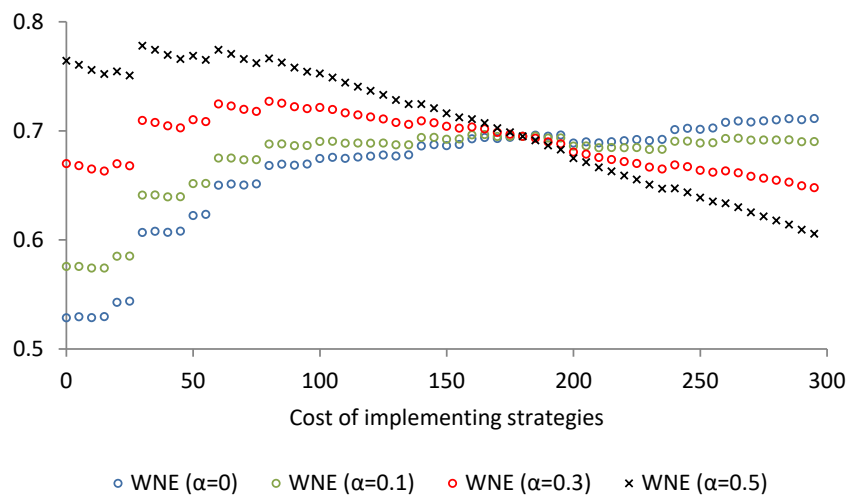

Figure 7: Variation of maximum weighted net evaluation $(W N E)$ of risk mitigation with different importance weights for cost $(\alpha)$ and mitigation cost.

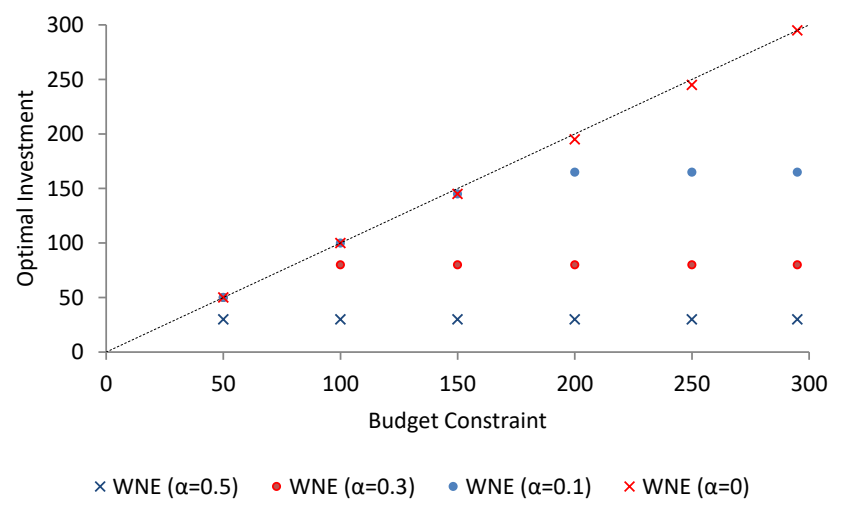

Figure 8: Optimal investment subject to different importance weights for cost $(\alpha)$ and budget constraint. 


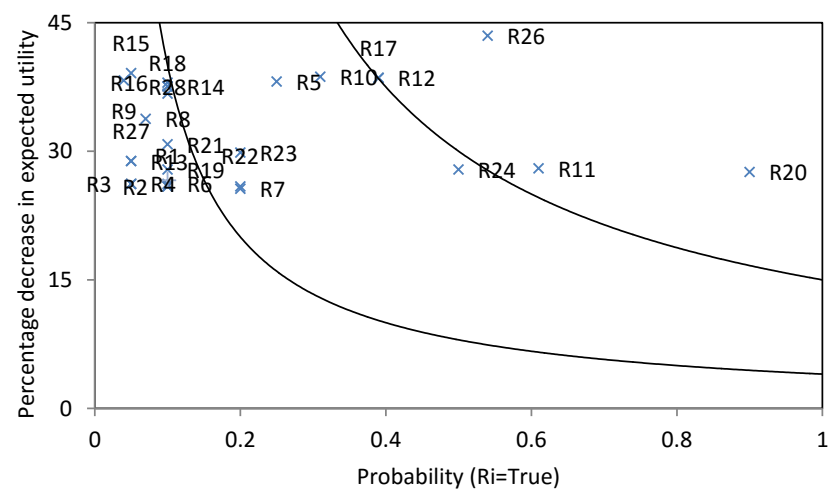

Figure 9: Risk matrix representing current state of risks (with no potential strategies implemented).

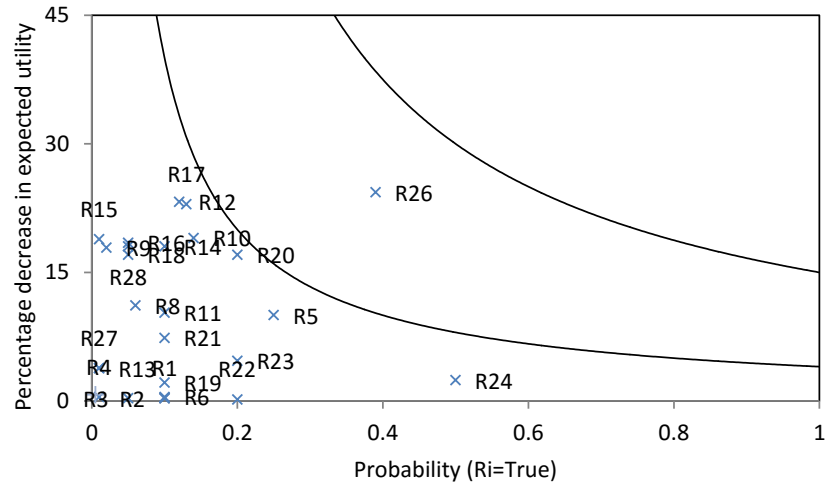

Figure 10: Risk matrix representing state of risks after implementation of the strategy "do everything" (all actions).

et al. (2015), this space can be partitioned into high, medium and low risk zones by using appropriate indifference curves. Implementing a risk mitigation strategy changes the location of risks as illustrated in Figure 10, and visualising the effect of a risk strategy on the location of those risks - in particular in relation to the critical thresholds - can assist decision-makers in reaching an optimal conclusion if such thresholds exist in the decision-maker's value system that are not captured by a simple expected utility measure.

\section{Discussion and Implications}

As the main aim of our research was to address two related questions, we discuss hereafter the implications of the research findings in order to explicitly address each question.

\subsection{A SCRM Process Integrating Interdependent Factors and the Risk Appetite}

In this section, we present a brief comparison of the proposed approach with the interdependency based approaches applied in other research areas and discuss the theoretical and managerial implications of the research.

RQ1: How can we develop and operationalise a SCRM process that captures interdependencies between risks, multiple (potentially conflicting) objectives (performance measures) and risk mitigation strategies specific to the risk appetite of a decision maker? 


\subsubsection{Comparison of the Proposed Approach with Interdependency Based Approaches in other Appli-}

cation Areas

With respect to interdependency modelling, there are a number of approaches (similar to the ones discussed in Section 2.2) applied to other application areas including but not limited to project risk management (Qazi et al., 2016; Zhang, 2016), enterprise resource planning (Aloini et al., 2012a b) and reliability of engineering systems (Ashrafi et al., 2015). Using the unique features of BBNs and EUT, the proposed approach not only captures probabilistic interdependency between risks but also integrates the risk appetite of a decision maker within the risk management process. We are not aware of any such risk management process especially in the literature on SCRM and project risk management that utilises the concept similar to WNE or introduces the 'probability-conditional expected utility' matrix for prioritising strategies and risks, respectively specific to the risk appetite of a decision maker.

Network theory and ISM based tools are useful in assessing the driving and dependency influence of risks (Aloini et al., 2012a b) whereas the proposed framework integrates these key features with the ability to model strength of interdependency between risks. The risk network provides an effective visual tool to help the decision maker prioritise risks on the basis of relative probability and propagation impact values thereby considering a holistic view of multiple factors including the position of a risk within the network, its influence on the key performance measures identified, and its probability of occurrence. The operationalisation scheme introduced in the paper provides an opportunity for researchers from diversified fields including but not limited to safety and reliability engineering, decision making under uncertainty, data analytics, and multi-criteria decision analysis to explore similar combinations of suitable techniques that could be adapted to the context of SCRM and easily adopted by practitioners.

\subsubsection{Theoretical Implications}

For better understanding, a block diagram is presented as Figure 11 which manifests the methodological contribution of this study to the established risk management process $(\mathrm{SA}, 2009)$. Although the application of the proposed process is demonstrated for a one-time decision problem of prioritising risks and mitigation strategies (at time: $T=t_{0}$ ), it can easily be extended to monitor and re-evaluate risks and strategies periodically. One of the key merits of the SCRNM process shown in Figure 3 is the operationalisation of each stage to help supply chain managers adopt an empirically tested technique for managing risks. This process also presents a unique integration of modelling interdependent risks, the decision maker's risk appetite and the trade-off across performance measures. There is a great deal of complexity associated with capturing the non-linear interactions between supply chain risks and performance measures, utility of the decision maker with regards to the tradeoff across these measures, and their risk appetite with regards to the cost and benefit of risk mitigation. For a detailed discussion on each stage of the risk management process, interested readers may consult SA $(2009)$. 


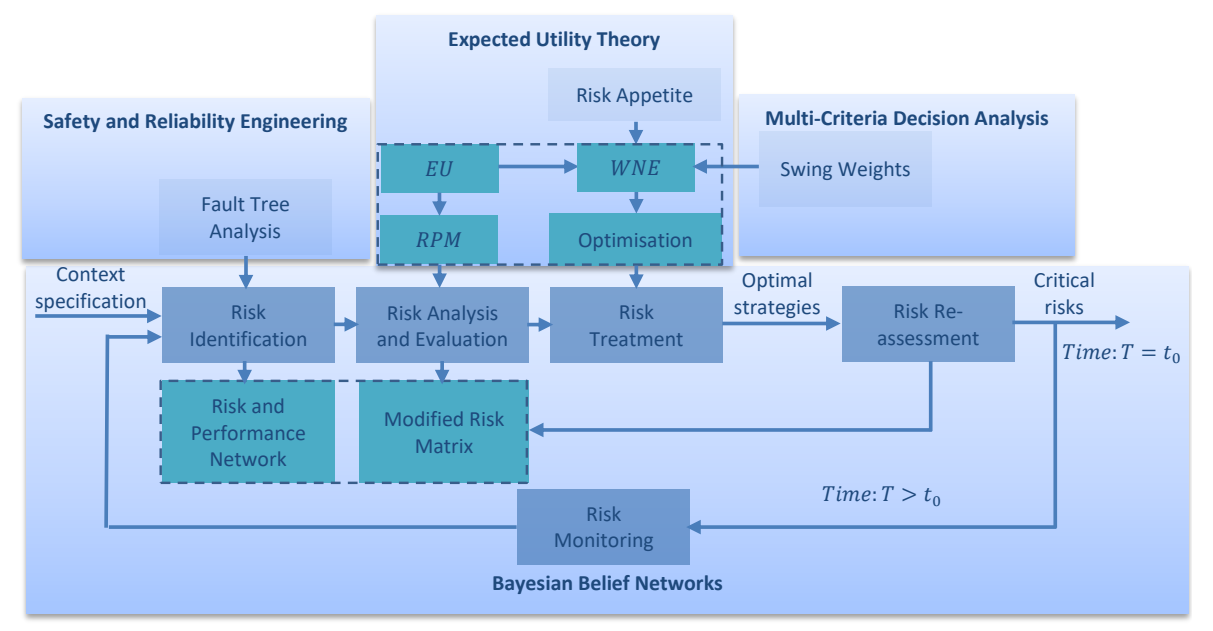

Figure 11: A block diagram representing the contribution of the proposed approach to the established risk management process $(\mathrm{SA}, 2009)$.

Risk Identification. Instead of following the conventional risk classification schemes the process introduces development of a risk and performance network where performance measures (objectives) are identified first followed by linking risks to these measures. Adopting such a technique (similar to the FTA) helps in not only modelling material risks but also common cause failures. The participants involved in developing the risk network were able to identify around 65 connections within the network. Furthermore, few risks located at the bottom of the network (business continuity management culture, risk management culture) were evaluated as critical risks having major influence on a number of risks. The selection of risks material to the performance measures identified is corroborated by Figure 9 where all risks possess higher values of conditional expected utility values representing the greater strength of interdependency within the risk network.

Risk Analysis. Risk matrix based tools and interdependency based models proposed in the literature generally focus on a single performance measure (monetary loss resulting from a risk realised) Garvey et al., 2015, Qazi et al. 2017) whereas it is important to consider all material performance measures including but not limited to quality, time, profit, competitive advantage, sustainability, cost and reputation. Instead of focussing on the monetary value of a loss resulting from a risk, the proposed process utilises the concept of conditional expected utility and each risk is evaluated with respect to its probability and influence on the overall expected utility across the risk network in terms of the risk propagation measure (see Equation (4). Instead of mapping each risk onto a probability-impact matrix (Khan et al., 2008; Duijm, 2015), the process introduces the 'probability-conditional expected utility' matrix thereby capturing the impact of each risk on all performance measures identified.

Risk Treatment and Risk Monitoring. In contrast with the treatment of individual risks and selection of individual risk specific strategies as followed in the conventional risk matrix based tools (Khan et al. 
2008; Tummala \& Schoenherr, 2011), the proposed process helps in mapping risk mitigation strategies

onto the risk network modelled. 'Weighted net evaluation of risk mitigation' and the method of 'swing weights' make it possible to establish the trade-off between the efficacy of potential risk mitigation strategies and the associated cost keeping in view the risk appetite of a decision maker. Ignoring the proposed process would increase the risk of selecting sub-optimal strategies. As the proposed process is grounded in the framework of BBNs, it is very easy to update the model once new risks are identified without the need for developing a new model from scratch. Similarly, a BBN based model can be easily maintained and monitored over a longer period to conduct a longitudinal study and systematically analyse important lessons learnt.

\subsubsection{Managerial Implications}

Based on the results of the case study, we introduce following propositions that will help the supply chain managers appreciate the significance of implementing a comprehensive interdependency based SCRM process.

Proposition 1. Neglecting interdependency between risks and strategies would risk the decision maker over- (under-) investing in implementing strategies.

In case of an exclusively independent supply risk network with no correlations between the risks, risk exposure is the summation of risk values corresponding to individual risks. Treating such independent risks with strategies influencing individual risks would yield a marginal benefit in terms of reducing (increasing) the overall risk exposure (expected utility). However, when there is a positive (negative) correlation between any single pair of risks a risk mitigation strategy targetting only one of these risks will generate a net reduction (increase) in the overall risk exposure that will be greater (smaller) than the case with no correlation between the risks. Therefore, a firm interested in achieving a specific risk exposure needs to invest less (more) if realisation of any risk triggers other risks and the strategies implemented have positive (negative) impact on the risk network.

For the risk network modelled (see Figure 44), optimal investment levels subject to different risk appetite and budget constraints are shown in Figure 8. Without following the proposed approach and ignoring interdependency between risks and strategies, the decision maker might select strategies that are optimal for individual risks, however, these might represent a sub-optimal choice (dominated combination of strategies) (see Figure 6). It is interesting to note that the impact of selecting suboptimal strategies is a non-linear function of investment level whereas the associated probability is a function of available combinations of strategies. The importance of this proposition was rightly acknowledged by one of our respondents:

"I think that the process helped us develop a risk network in a very short time and it was quite helpful to think through developing the network from the performance measures. We could identify 
some interesting patterns and specifically the identification of sub-optimal strategies through the process

is fascinating and worth investing time and effort. I am sure that such decision tools will add real value to any enterprise and help them make efficient investment decisions" (Resp\#1).

Proposition 2. The upper (lower) bound of the expected utility value corresponding to the set of objectives modelled within a risk network is determined by the efficacy of potential (already implemented) risk mitigation strategies in reducing the risk level of related risks.

All performance measures are not necessarily equally important to the decision maker (Jüttner et al. 2003). When these performance measures are treated in isolation and independently optimised, the resulting strategies might not yield a global optimal solution corresponding to the holistic interaction of these measures within the network setting. Expected utility is a probability-weighted average of the utility in the different states the network may be in. By engaging in risk mitigation, the probability of these states occurring changes, as does the value of the objectives. More generally, a utility function could capture different weights being assigned to different objectives, objectives may be evaluated in a non-linear way, and complementarities between objectives could be captured.

The lower bound of the expected utility reflects the efficacy of already implemented strategies as to how comfortable the decision maker is with regard to the current state of the risk management process. With reference to the model developed in the case study, the point corresponding to the mitigation cost of 0 represents the efficacy of already implemented strategies with the global minimum expected utility value of 0.528 (see Figure 6). This lower bound can further drop in the event of unfavorable correlations within the network. However, as the proposed risk mitigation strategies were negatively correlated with all risks, the expected utility of the network would not reduce below 0.528. The upper bound of the expected utility is determined by the efficacy of potential strategies, however, there is another constraint of the budget and the need for an important consideration as to the significance of the relative improvement in expected utility with respect to the marginal cost of implementing these strategies. Although an investment of 295 units yields the highest expected utility to the decision maker (see Figure 6), the same is not viable considering the cost-effectiveness of strategies (see Figure 8). The trade-off between the reduction in risk exposure and the cost involved in implementing strategies is governed by the weight the decision-maker attached to the cost (see Equation 5). The participants involved in the case study were able to link this proposition to the significance of adopting the proposed process as it is not possible to ascertain these limits using traditional risk matrix based models and also, the performance of the risk management process could be benchmarked against these limits, once established.

Proposition 3. Even in the case of all risks being positively correlated with each other and strategies negatively correlated with risks, increased investment in strategies might not necessarily increase the expected utility of the decision maker. 
Keeping in view a given set of potential risk mitigation strategies with associated cost, there are

different possible combinations of strategies subject to a budget constraint with only one optimal combination (see Figure 6). However, with the increase in the budget constraint, it is not always the case that the new optimal combination contains all strategies included in the optimal set previously determined subject to a lower constraint that could lead to a reduction in the expected utility (see the budget range of $195-235$ in Figure 6). Therefore, there is always a need for analysing a complete portfolio of all such combinations of strategies rather than evaluating the strategies at the given constraint only. The optimistic viewpoint with regards to the favourable correlations between risks and strategies might be misleading as a supply chain manager would incorrectly assume that investing in additional strategies and choosing the optimal combination right at the constraint level is viable. The supply chain risk managers involved in our case study appreciated the fact that without using the proposed modelling approach, they would not be able to realise that it was not worth investing in strategies representing the budget range of $195-235$. Related to this, they were able to identify all such combinations of sub-optimal strategies.

Proposition 4. Within a network setting and in the case of partially effective risk mitigation strategies, it is not always optimal to mitigate the most critical risk(s) identified; instead strategies implemented for relatively non-critical risk(s) might be cost-effective.

It is very important to realise that within a risk management framework with interdependence (see Figure 3), risk prioritisation follows the risk treatment stage in contrast to the sequence proposed in the standard risk management framework $(\overline{\mathrm{SA}}, 2009)$ and established SCRM frameworks (Manuj \& Mentzer, 2008, Tummala \& Schoenherr, 2011; Giannakis \& Papadopoulos, 2016). This is because of the complexity involved in evaluating the efficacy of strategies that is a function of the strength of interdependency between risks, the relative impact of strategies, the cost of these strategies and the relative importance of performance measures influenced by the risks. Therefore, implementing costeffective strategies might not necessarily reduce the most critical risks substantially and that is why the risk assessment must follow the risk treatment stage to prioritise risks for the risk monitoring stage and developing contingency plans.

With reference to the model developed, although $R 20$ is evaluated as a critical risk during the risk assessment stage (see Figure 9), it is not optimal to adopt the relevant strategy subject to a budget constraint of 30 units (see Figure 6 and Table A.5p. This is because the optimal set comprises two costeffective mitigation strategies applied to relatively less critical risks ( $R 4$ and $R 12$ ) yielding maximum expected utility to the decision maker whereas exclusively mitigating $R 20$ is the most expensive option (costing 100 units) among the set of potential strategies (see Table A.5). Therefore, it is not always optimal to mitigate the most critical risk(s) identified. Adopting a risk matrix based approach would fail to capture the complex dynamics between risks and strategies considering the cost of strategies 
and relative importance of each performance measure. This proposition is also substantiated through

comparing Figure 9 and Figure 10 where $R 26$ remains a critical risk even after implementing all strategies. Hence, the implementation of potential strategies does not necessarily mitigate risks in their order of criticality rather the significance of these strategies is a function of their relative position in the network, associated cost, risk mitigation effectiveness and the decision maker's preference in reducing the cost adjusted risk exposure.

\subsection{Merits and Challenges}

In this section, we present our findings about the current practices followed in the industry and describe the merits and challenges associated with the implementation of the proposed process. Sample quotations from the interview narratives are presented in Appendix B.

RQ2: What are the merits and challenges associated with the implementation of the proposed process?

\subsubsection{Current Practices}

The main aim of the study was to develop and empirically evaluate an integrated SCRM process and to investigate the merits and challenges associated with implementing the process. The case study helped us gain an insight into the real practices of managing supply chain risks. Our findings conform to the widely reported literature on supply chain risk classification where risks are classified into independent categories and on risk management frameworks where the notion of assessing these risks in silo is embraced (Rangel et al. 2015). Limited tools or techniques focussing on the interdependency modelling are confined to optimising a single performance measure and therefore, the optimisation of these measures in isolation does not necessarily yield a global optimal solution (Colicchia \& Strozzi, 2012, Garvey et al. 2015). This finding corroborates the study conducted by Ho et al. (2015) who have emphasised the need for integrating all stages of the risk management process and linking systemic risks to (potentially conflicting) objectives. The participants also echoed the same concern and acknowledged the limitation of existing practices.

\subsubsection{Merits of the Proposed Process}

The participants involved in the case study found it a very interesting exercise to develop a risk network and link risks to multiple performance measures. Related to this, the use of an approach similar to the FTA was highly appreciated as it would ensure focussing on important risks only and not considering risks having insignificant impact on a performance measure. To the best of the authors' knowledge, there are limited studies having explored the use of FTA in modelling supply chain risks (Sherwin et al., 2016). However, merging the two techniques of FTA and BBNs helps in modelling common cause failures that cannot be achieved through the use of FTA alone. 
The elicitation of conditional probability values was easier in the case of experts having background

knowledge in engineering or mathematical science whereas it was a challenging task otherwise. The use of a qualitative scale helped the experts provide their judgement and with the passage of time, they were able to indicate numeric values. The participants could appreciate the significance of optimising conflicting objectives within the same model. The main merit of the proposed process was acknowledged as the ability to visualise the interconnectedness between the risks and how exactly a risk or a set of risks influences multiple objectives. Techniques other than BBNs are not able to depict the similar kind of transparency and visual patterns of risk propagation (Garvey et al., 2015). The graphs representing the efficacy of potential risk mitigation strategies were highly appreciated as these helped the decision makers realise the significance of adopting the proposed process without which it would not be possible to segregate optimal strategies from the dominated ones.

Another key feature of the process acknowledged by the participants relates to capturing both the trade-off between difference performance measures and the influence of interdependent risks on these measures as one of the respondents mentioned that: "We do not have tools to model the trade-off across the objectives within an interdependent setting of interacting risks. If you do not map the main sources of risks to your performance and if you do not map the correlations then you can have a serious problem and now we are able to identify the main limitation of our current process" (Resp\#4). As the organisation studied currently utilises a risk matrix based tool, the proposed 'probability-conditional expected utility' risk matrix was considered a very relevant and significant contribution. Substantiating the utility of the proposed process, the participants realised that soft factors like corporate governance and risk management culture, and unquantifiable risks like regulatory changes and reputational damage could easily be captured in the modelling process as there is no such requirement of establishing risk specific monetary loss values.

\subsubsection{Challenges Associated with the Adoption of Proposed Process}

Despite acknowledging the merits of the proposed process, the participants were apprehensive of the challenges involved. As the risk management process is often governed by regulations, the established frameworks like SA (2009) with their exclusive focus on evaluating and managing individual risks would need to be challenged and replaced by interdependency based frameworks. The second major problem relates to the organisational culture and the resistance to change. The development of SCRM in theory dates back to the start of 21 st century (Manuj \& Mentzer, 2008) and therefore, it is too early for practitioners to realise and implement the risk management process in its true essence. It needs a lot of commitment from the top management to indoctrinate the culture of risk management as practitioners mostly rely on their intuition and past experiences and they tend to be reluctant to change their practices. Also, it needs a lot of effort in terms of educating the people involved and maintaining such models over a period of time. 


\subsection{Limitations and Future Research Directions}

Studies focussing on the cost and benefit analysis of implementing these sophisticated frameworks would incentivise practitioners towards adopting interdependency modelling in managing risks. Like advocated by Ackermann \& Alexander (2016), we think that there is a need for "finding mechanisms to encourage the application of the (interdependency based) approach" (Ackermann \& Alexander, 2016. p. 899) by SCRM professionals. Similarly, the dynamic nature of risk could be captured as the risk networks "created at a particular point in time could be compared with those of a later time period thus enabling longitudinal analysis of projects, allowing for shifting patterns of behaviour to be explored" (Ackermann \& Alexander, 2016, p. 899).

Although risk matrix based tools have been criticised for their inherent limitations, there is a great opportunity to improve these mainly because of their popularity with practitioners (Duijm, 2015). In the proposed process, we improve the conventional risk matrix in terms of capturing the network-wide impact of individual risks. However, it might be interesting to develop such maps to reflect systemic interaction between risks and project multiple risk scenarios. Furthermore, conventional tools must be further developed to capture the dynamic nature of risk. As the risk network developed does not cover all aspects of the supply chain including engineering facets mainly because of the main intention of demonstrating the proposed process and not developing a comprehensive risk network, future research may be directed to developing such risk networks specific to different industries. This will help in developing a risk network based taxonomy and understanding common patterns of risk paths.

We have mainly used BBNs to model interdependency between risks, it is worth integrating a number of techniques feasible for each stage of the process and validating the efficacy of the resulting hybrid risk management process through case studies. Algorithms can be developed to establish the efficacy of such integrated tools considering the effort involved and the precision of results obtained. The case study presented was a one-time demonstration of the proposed process whereas it is important to explore the long-term implications of adopting the process through conducting longitudinal case studies. For simplicity of exposition we have treated risks as binary variables, and it would be interesting to extend this in a meaningful way to more general characterisations of risk. It would also be interesting to consider extending the framework to account for different stakeholders across a supply chain that may have conflicting objectives and conceptualise contractual frameworks to encourage active participation of stakeholders within the risk management process.

\section{Conclusions}

Although a number of quantitative tools and techniques have already been developed for managing supply chain risks, there is a limited focus on introducing holistic frameworks that not only integrate all stages of the risk management process but also capture the cascading effects of common risk triggers and 
the risk appetite of a decision maker. Also, existing frameworks generally focus on optimising a single

objective (performance measure) without exclusively modelling the trade-off between conflicting and interdependent objectives. Another important requirement is to empirically evaluate these frameworks and establish the merits and challenges involved in implementing such interdependency based tools. In order to bridge the mentioned gaps, we have developed and operationalised an integrated SCRNM process, and conducted a case study to demonstrate its application. Established techniques from safety and reliability engineering, decision making under uncertainty and multi-criteria decision analysis were adapted and integrated together to operationalise the proposed process.

The organisation studied exclusively utilises risk matrix based tools to assess risks. As conceptualised in the literature, risks are classified into independent categories and correlations are neglected in all stages of the risk management process. Such assumptions are deleterious to the main objective of implementing an effective process as the risk health of a supply chain might be suboptimal if interdependencies in the risk network are ignored. Developing a risk network originating from the performance measures helps in confining the scope to significant risks only and therefore, risks having insignificant impact on the measures are not considered. The risk network also helps in identifying potential mitigation strategies and establishing their correlations with relevant risks.

The practitioners adhere to using conventional tools treating risks as independent factors because of various reasons: sophisticated interdependency based tools introduced in theory are rarely applied in industry; practitioners are unable to appreciate the significance of capturing correlations until they acknowledge the extent of damage relevant to adopting risk matrix based tools; use of risk matrix is governed by established risk management standards; and there is not always a commitment from the top management as the implementation of a robust process necessitates time and investment in terms of training staff and enhancing their knowledge to assimilate the underlying mechanism of the process.

There are some limitations of our study: we have only conducted a single case study and not involved other stakeholders of the supply chain; the risk network developed does not cover all aspects of the supply chain as the main aim was to demonstrate the application of the proposed process and therefore, not all categories of supply chain risks are considered; we have captured one-time risk state of the risk network rather than monitoring the dynamic nature of the risk; risks and mitigation strategies are modelled as binary variables; and conflicting incentives of main stakeholders are not modelled. The research work can be developed further along different lines of inquiry. The efficacy of the proposed process may be monitored over a period of time through a longitudinal study and the merits and challenges analysed. The framework may be extended to involve different stakeholders across the supply chain and contracts be designed to encourage active participation of the stakeholders. Comprehensive risk networks may be developed across different industries and compared to establish common patterns in order to develop a generalised risk network based taxonomy. The cost and benefit analysis may be 
conducted to help practitioners understand the utility of interdependency based frameworks with regard

to the resources dedicated to a similar process and the benefits resulting from its implementation. Once the framework gets established in its simplified form of risks and strategies with binary states, more general characterisation of risks can be captured. The framework may also be extended to capture the dynamic behavior of risk over time.

\section{Acknowledgements}

This research has been supported by the Mac Robertson Scholarship awarded to the first author. The authors wish to thank the editor and anonymous reviewers for their constructive remarks that significantly helped the improvement of this paper.

\section{References}

Ackermann, F., \& Alexander, J. (2016). Researching complex projects: Using causal mapping to take a systems perspective. International Journal of Project Management, 34, 891-901.

Ackermann, F., Howick, S., Quigley, J., Walls, L., \& Houghton, T. (2014). Systemic risk elicitation: Using causal maps to engage stakeholders and build a comprehensive view of risks. European Journal of Operational Research, 238, 290-299.

Aloini, D., Dulmin, R., \& Mininno, V. (2012a). Modelling and assessing erp project risks: A petri net approach. European Journal of Operational Research, 220, 484-495.

Aloini, D., Dulmin, R., \& Mininno, V. (2012b). Risk assessment in erp projects. Information Systems, 37, 183-199.

Aqlan, F., \& Lam, S. S. (2015). Supply chain risk modelling and mitigation. International Journal of Production Research, 53, 5640-5656.

Ashrafi, M., Davoudpour, H., \& Khodakarami, V. (2015). Risk assessment of wind turbines: Transition from pure mechanistic paradigm to modern complexity paradigm. Renewable and Sustainable Energy Reviews, 51, 347-355.

Aven, T. (2015). Risk assessment and risk management: Review of recent advances on their foundation. European Journal of Operational Research, 253, 1-13.

Aven, T., \& Kristensen, V. (2005). Perspectives on risk: review and discussion of the basis for establishing a unified and holistic approach. Reliability Engineering $\mathcal{G}$ System Safety, 90, 1-14. 
Badurdeen, F., Shuaib, M., Wijekoon, K., Brown, A., Faulkner, W., Amundson, J., Jawahir, I. S.,

Goldsby, T. J., Iyengar, D., \& Boden, B. (2014). Quantitative modeling and analysis of supply chain risks using bayesian theory. Journal of Manufacturing Technology Management, 25, 631-654.

Belton, V., \& Stewart, T. J. (2002). Value function methods: Practical basics. In Multiple Criteria Decision Analysis: An Integrated Approach (pp. 119-161). Boston, MA: Springer US.

Blos, M. F., \& Miyagi, P. E. (2015). Modeling the supply chain disruptions focusing on the supply chain interdependencies. In 2015 IEEE 13th International Conference on Industrial Informatics (INDIN) (pp. 604-609).

Christopher, M., Mena, C., Khan, O., \& Yurt, O. (2011). Approaches to managing global sourcing risk. Supply Chain Management: An International Journal, 16, 67-81.

Christopher, M., \& Peck, H. (2004). Building the resilient supply chain. International Journal of Logistics Management, 15, 1-14.

Colicchia, C., \& Strozzi, F. (2012). Supply chain risk management: a new methodology for a systematic literature review. Supply Chain Management: An International Journal, 17, 403-418.

Dogan, I., \& Aydin, N. (2011). Combining bayesian networks and total cost of ownership method for supplier selection analysis. Computers and Industrial Engineering, 61, 1072-1085.

Dong, Q., \& Cooper, O. (2016). An orders-of-magnitude $\{$ AHP $\}$ supply chain risk assessment framework. International Journal of Production Economics, 182, 144 - 156.

Duijm, N. J. (2015). Recommendations on the use and design of risk matrices. Safety Science, 76 , $21-31$.

Elleuch, H., Hachicha, W., \& Chabchoub, H. (2014). A combined approach for supply chain risk management: description and application to a real hospital pharmaceutical case study. Journal of Risk Research, 17, 641-663.

Faisal, M. N., Banwet, D. K., \& Shankar, R. (2006). Supply chain risk mitigation: modeling the enablers. Business Process Management Journal, 12, 535-552.

Ganguly, K. (2014). Integration of analytic hierarchy process and dempster-shafer theory for supplier performance measurement considering risk. International Journal of Productivity and Performance Management, 63, 85-102.

Garvey, M. D., Carnovale, S., \& Yeniyurt, S. (2015). An analytical framework for supply network risk propagation: A bayesian network approach. European Journal of Operational Research, 243, 618-627. 
Gaudenzi, B., \& Borghesi, A. (2006). Managing risks in the supply chain using the ahp method.

International Journal of Logistics Management, 17, 114-136.

GeNIe (2.0). The decision systems laboratory, genie and smile. retrieved 5 june, 2014, from http://genie.sis.pitt.edu/about.html. URL: http://genie.sis.pitt.edu/about.html.

Ghadge, A., Dani, S., Chester, M., \& Kalawsky, R. (2013). A systems approach for modelling supply chain risks. Supply Chain Management: An International Journal, 18, 523-538.

Giannakis, M., \& Papadopoulos, T. (2016). Supply chain sustainability: A risk management approach. International Journal of Production Economics, 171, Part 4, 455-470.

Gillham, B. (2000). Case Study Research Methods. Bloomsbury Academic.

Giunipero, L. C., \& Eltantawy, R. A. (2004). Securing the upstream supply chain: a risk management approach. International Journal of Physical Distribution 8 Logistics Management, 34, 698-713.

Hallikas, J., Karvonen, I., Pulkkinen, U., Virolainen, V.-M., \& Tuominen, M. (2004). Risk management processes in supplier networks. International Journal of Production Economics, 90, 47-58.

Harland, C., Brenchley, R., \& Walker, H. (2003). Risk in supply networks. Journal of Purchasing and Supply Management, 9, 51-62.

Heckmann, I., Comes, T., \& Nickel, S. (2015). A critical review on supply chain risk -definition, measure and modeling. Omega, 52, 119-132.

Ho, W., Zheng, T., Yildiz, H., \& Talluri, S. (2015). Supply chain risk management: a literature review. International Journal of Production Research, 53, 5031-5069.

Hosseini, S., \& Barker, K. (2016). A bayesian network model for resilience-based supplier selection. International Journal of Production Economics, 180, 68-87.

Jensen, F. V., \& Nielsen, T. D. (2007). Bayesian networks and decision graphs. Springer-Verlag, New York.

Jüttner, U., Peck, H., \& Christopher, M. (2003). Supply chain risk management: outlining an agenda for future research. International Journal of Logistics Research and Applications, 6, 197-210.

Kelangath, S., Das, P. K., Quigley, J., \& Hirdaris, S. E. (2011). Risk analysis of damaged ships -a data-driven bayesian approach. Ships and Offshore Structures, 7, 333-347.

Khan, O., Christopher, M., \& Burnes, B. (2008). The impact of product design on supply chain risk: a case study. International Journal of Physical Distribution \& Logistics Management, 38, 412-432. 
Kim, Y., Choi, T. Y., Yan, T., \& Dooley, K. (2011). Structural investigation of supply networks: A social network analysis approach. Journal of Operations Management, 29, 194-211.

Kleindorfer, P. R., \& Saad, G. H. (2005). Managing disruption risks in supply chains. Production and Operations Management, 14, 53-68.

Knemeyer, A. M., Zinn, W., \& Eroglu, C. (2009). Proactive planning for catastrophic events in supply chains. Journal of Operations Management, 27, 141-153.

Lavastre, O., Gunasekaran, A., \& Spalanzani, A. (2012). Supply chain risk management in french companies. Decision Support Systems, 52, 828-838.

Leerojanaprapa, K. (2014). A Bayesian belief network modelling process for systemic supply chain risk. Ph.D. thesis University of Strathclyde.

Leerojanaprapa, K., van der Meer, R., \& Walls, L. (2013). Modeling supply risk using belief networks: A process with application to the distribution of medicine. In IEEE International Conference on Industrial Engineering and Engineering Management (pp. 201-205).

Lin, Y., \& Zhou, L. (2011). The impacts of product design changes on supply chain risk: a case study. International Journal of Physical Distribution \& Logistics Management, 41, 162-186.

Lockamy, A., \& McCormack, K. (2009). Analysing risks in supply networks to facilitate outsourcing decisions. International Journal of Production Research, 48, 593-611.

Manuj, I., \& Mentzer, J. T. (2008). Global supply chain risk management. Journal of Business Logistics, 29, 133-155.

Micheli, G. J. L., Mogre, R., \& Perego, A. (2014). How to choose mitigation measures for supply chain risks. International Journal of Production Research, 52, 117-129.

Nadkarni, S., \& Shenoy, P. P. (2004). A causal mapping approach to constructing bayesian networks. Decision Support Systems, 38, 259-281.

Nepal, B., \& Yadav, O. P. (2015). Bayesian belief network-based framework for sourcing risk analysis during supplier selection. International Journal of Production Research, 53, 6114-6135.

Norrington, L., Quigley, J., Russell, A., \& Van der Meer, R. (2008). Modelling the reliability of search and rescue operations with bayesian belief networks. Reliability Engineering E System Safety, 93, 940-949.

Norrman, A., \& Jansson, U. (2004). Ericsson's proactive supply chain risk management approach after a serious sub-supplier accident. International Journal of Physical Distribution 83 Logistics Management, $34,434-456$. 
Oehmen, J., Ziegenbein, A., Alard, R., \& Schonsleben, P. (2009). System-oriented supply chain risk management. Production Planning \& Control, 20, 343-361.

Pfohl, H.-C., Gallus, P., \& Thomas, D. (2011). Interpretive structural modeling of supply chain risks. International Journal of Physical Distribution 83 Logistics Management, 41, 839-859.

Pitchforth, J., \& Mengersen, K. (2013). A proposed validation framework for expert elicited bayesian networks. Expert Systems with Applications, 40, 162-167.

Qazi, A., Quigley, J., Dickson, A., \& Ekici, S. O. (2017). Exploring dependency based probabilistic supply chain risk measures for prioritising interdependent risks and strategies. European Journal of Operational Research, 259, 189-204.

Qazi, A., Quigley, J., Dickson, A., \& Kirytopoulos, K. (2016). Project complexity and risk management (procrim): Towards modelling project complexity driven risk paths in construction projects. International Journal of Project Management, 34, 1183-1198.

Rangel, D. A., de Oliveira, T. K., \& Leite, M. S. A. (2015). Supply chain risk classification: discussion and proposal. International Journal of Production Research, 53, 6868-6887.

Ritchie, B., \& Brindley, C. (2007). An emergent framework for supply chain risk management and performance measurement. The Journal of the Operational Research Society, 58, 1398-1411.

Rotaru, K., Wilkin, C., \& Ceglowski, A. (2014). Analysis of scor's approach to supply chain risk management. International Journal of Operations \& Production Management, 34, 1246-1268.

Ruan, X., Yin, Z., \& Frangopol, D. M. (2015). Risk matrix integrating risk attitudes based on utility theory. Risk Analysis, 35, 1437-1447.

SA (2009). Risk Management: Principles and Guidelines (AS/NZS ISO 31000: 2009). Standards Australia, Sydney.

Sherwin, M. D., Medal, H., \& Lapp, S. A. (2016). Proactive cost-effective identification and mitigation of supply delay risks in a low volume high value supply chain using fault-tree analysis. International Journal of Production Economics, 175, 153-163.

Sigurdsson, J. H., Walls, L. A., \& Quigley, J. L. (2001). Bayesian belief nets for managing expert judgement and modelling reliability. Quality and Reliability Engineering International, 17, 181-190.

Sinha, P. R., Whitman, L. E., \& Malzahn, D. (2004). Methodology to mitigate supplier risk in an aerospace supply chain. Supply Chain Management: An International Journal, 9, 154-168. 
Tang, C., \& Tomlin, B. (2008). The power of flexibility for mitigating supply chain risks. International

Journal of Production Economics, 116, 12-27.

Tang, C. S. (2006). Perspectives in supply chain risk management. International Journal of Production Economics, 103, 451-488.

Tang, O., \& Nurmaya Musa, S. (2011). Identifying risk issues and research advancements in supply chain risk management. International Journal of Production Economics, 133, 25-34.

Tummala, R., \& Schoenherr, T. (2011). Assessing and managing risks using the supply chain risk management process (scrmp). Supply Chain Management: An International Journal, 16, 474-483.

Tuncel, G., \& Alpan, G. (2010). Risk assessment and management for supply chain networks: A case study. Computers in Industry, 61, 250-259.

Wilson, K. J., \& Quigley, J. (2016). Allocation of tasks for reliability growth using multi-attribute utility. European Journal of Operational Research, 255, 259-271.

Wu, W.-S., Yang, C.-F., Chang, J.-C., Chateau, P.-A., \& Chang, Y.-C. (2015). Risk assessment by integrating interpretive structural modeling and bayesian network, case of offshore pipeline project. Reliability Engineering 8 System Safety, 142, 515-524.

Yin, R. K. (2009). Case Study Research: Design and Methods volume 5. (4th ed.). SAGE Publications. Zhang, Y. (2016). Selecting risk response strategies considering project risk interdependence. International Journal of Project Management, 34, 819-830.

Zhao, L., Huo, B., Sun, L., \& Zhao, X. (2013). The impact of supply chain risk on supply chain integration and company performance: a global investigation. Supply Chain Management: An International Journal, 18, 115-131. 
Appendix A.

\begin{tabular}{|c|c|c|c|c|}
\hline Article & $\begin{array}{c}\text { Interdependency } \\
\text { modelling of } \\
\text { risks }\end{array}$ & $\begin{array}{c}\text { Risk } \\
\text { Appetite }\end{array}$ & $\begin{array}{l}\text { Interdependency } \\
\text { between risks and } \\
\text { strategies }\end{array}$ & $\begin{array}{l}\text { Qualitative (*)/ } \\
\text { Quantitative (o) }\end{array}$ \\
\hline Harland et al. (2003) & & & & $*$ \\
\hline Hallikas et al. (2004) & & & & $*$ \\
\hline Giunipero and Eltantawy (2004) & & & & $*$ \\
\hline Norrman and Jansson (2004) & & & & Semi-quantitative \\
\hline Sinha et al. (2004) & & & & $*$ \\
\hline Kleindorfer and Saad (2005) & & & & Semi-quantitative \\
\hline Khan et al. (2008) & & & & $*$ \\
\hline Manuj and Mentzer (2008) & & & $x$ & $*$ \\
\hline Knemeyer et al. (2009) & & $x$ & $x$ & Semi-quantitative \\
\hline Oehmen et al. (2009) & $x$ & & $x$ & $*$ \\
\hline Tuncel and Alpan (2010) & & & $x$ & o \\
\hline Tummala and Schoenherr (2011) & & & & $*$ \\
\hline Lavastre et al. (2012) & & $x$ & & Semi-quantitative \\
\hline Elleuch et al. (2014) & & & $x$ & 0 \\
\hline Rotaru et al. (2014) & & & & $*$ \\
\hline Micheli et al. (2014) & & & $x$ & 0 \\
\hline Aqlan and Lam (2015) & & & $x$ & 0 \\
\hline Giannakis and Papadopoulos (2016) & $x$ & & & Semi-quantitative \\
\hline Qazi et al. (2017) & $\mathrm{x}$ & & $x$ & 0 \\
\hline
\end{tabular}

Table A.1: Selected articles with focus on different research themes.

\begin{tabular}{lcc}
\hline \multicolumn{1}{c}{ Designation } & $\begin{array}{c}\text { Work experience } \\
\text { (no. of years) }\end{array}$ & Respondent ID \\
\hline Risk Manager & 19 & Resp\#1 \\
Purchasing and Supply Chain Manager & 25 & Resp\#2 \\
Loss Prevention Analyst & 5 & Resp\#3 \\
Insurance Manager & 13 & Resp\#4 \\
Project Manager & 20 & Resp\#5 \\
Project Risk Manager & 15 & Resp\#6 \\
Project Risk Manager & 16 & Resp\#7 \\
\hline
\end{tabular}

Table A.2: Profile of respondents (semi-structured interviews). 


\begin{tabular}{lcc}
\hline \multicolumn{1}{c}{ Risk/Risk Source } & States & ID \\
\hline Unexpected event (Supplier) & Yes, No & R1 \\
Unexpected event (Aero) & Yes, No & R2 \\
Information and Communication Technology (ICT) System disruption & Yes, No & R3 \\
(Supplier) & Yes, No & R4 \\
ICT System disruption (Aero) & Bad, Good & R5 \\
Corporate governance & Yes, No & R6 \\
Regulatory changes & Low, High & R7 \\
Investment in loss prevention and sustainability & Yes, No & R8 \\
Labour related diseases & Yes, No & R9 \\
Fatal accident & Yes, No & R10 \\
Breaking code of conduct & Bad, Good & R11 \\
Business continuity management culture & Bad, Good & R12 \\
Risk management culture & Yes, No & R13 \\
Strikes (Aero) & Yes, No & R14 \\
Strikes (Supplier) & Yes, No & R15 \\
Lack of control (Aero) & Yes, No & R16 \\
Lack of control (Supplier) & Yes, No & R17 \\
Lack of procedures (Aero) & Yes, No & R18 \\
Lack of procedure (Supplier) & Yes, No & R19 \\
Logistics problems & High, Low & R20 \\
Aero price vs. Competitor price & Yes, No & R21 \\
Supplier problems with environmental, health and safety (EHS) & Ineffective, & R22 \\
Communication plan & Effective & \\
Change in specification by customer & Yes, No & R23 \\
Customer pressure on delivery & Yes, No & R24 \\
Financial issues & Yes, No & R25 \\
Aero quality vs. Competitor quality & Low, High & R26 \\
Human error (Aero) & Yes, No & R27 \\
Human error (Supplier) & Yes, No & R28 \\
\hline & & \\
& &
\end{tabular}

Table A.3: Risks and risk sources considered in the modelling framework.

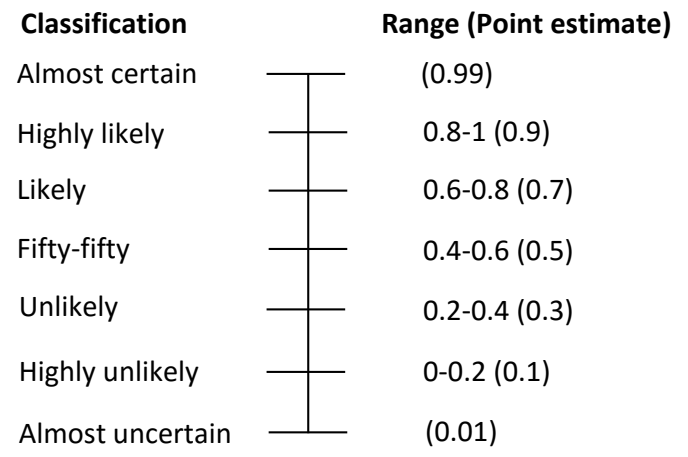

Figure A.1: Scale used for eliciting probability values. 
Maximum

(1)

Very high

High

Medium

Low

Very low

Minimum

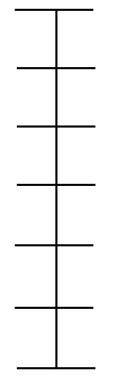

0.8-1 (0.9)

0.6-0.8 (0.7)

0.4-0.6 (0.5)

0.2-0.4 (0.3)

0-0.2 (0.1)

(0)

Figure A.2: Scale used for eliciting utility values.
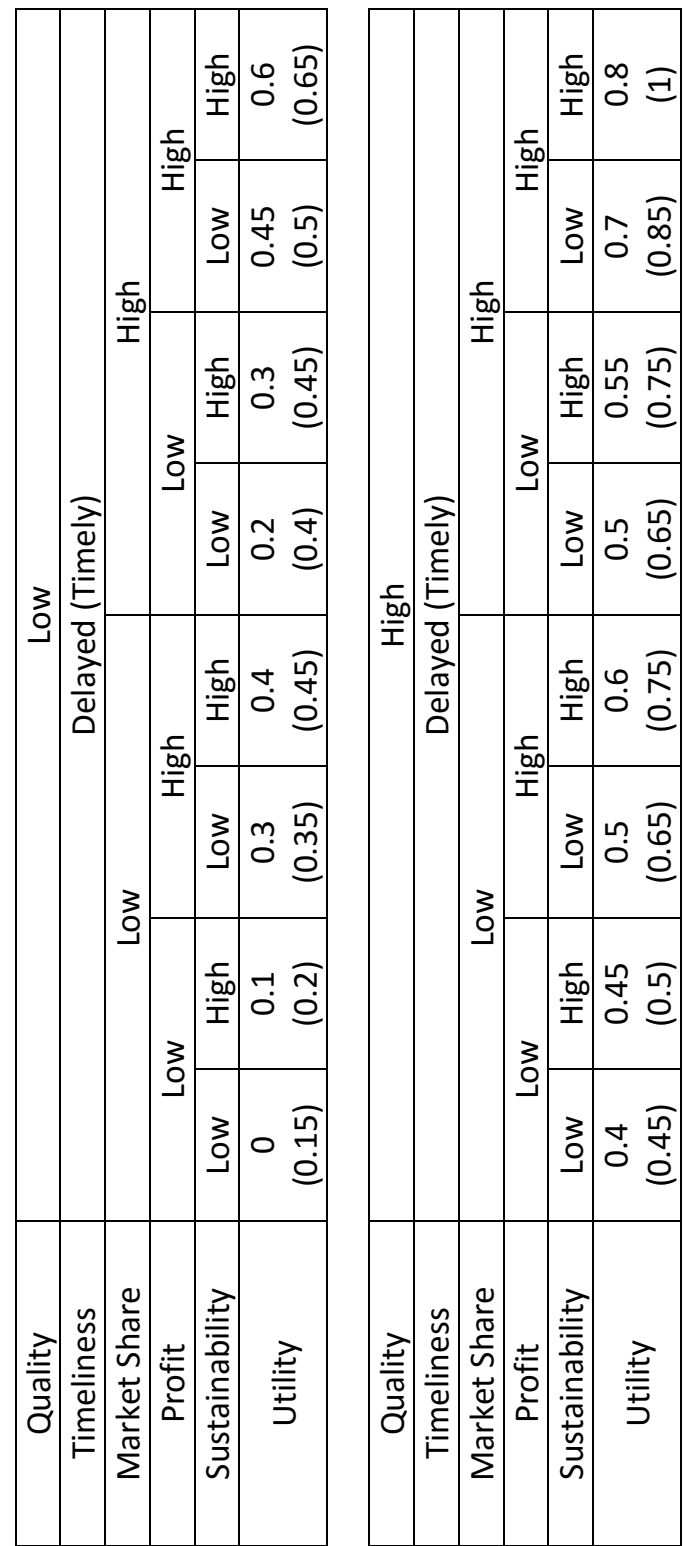


$\begin{array}{ll}\text { Contract Terms } & 20 \\ & 20\end{array}$

Quality Training $\quad 20$

Perform Business Interruption Analysis $\quad 20$

Adopt Enterprise Risk Management Model $\quad 30$

Perform Disaster Recovery Plan (DRP) Testing $\quad 10$

Union Relations 5

Economies of Scale $\quad 30$

Flexibility $\quad 60$

Reduce Cost 100

Table A.5: Potential risk mitigation strategies and associated cost.

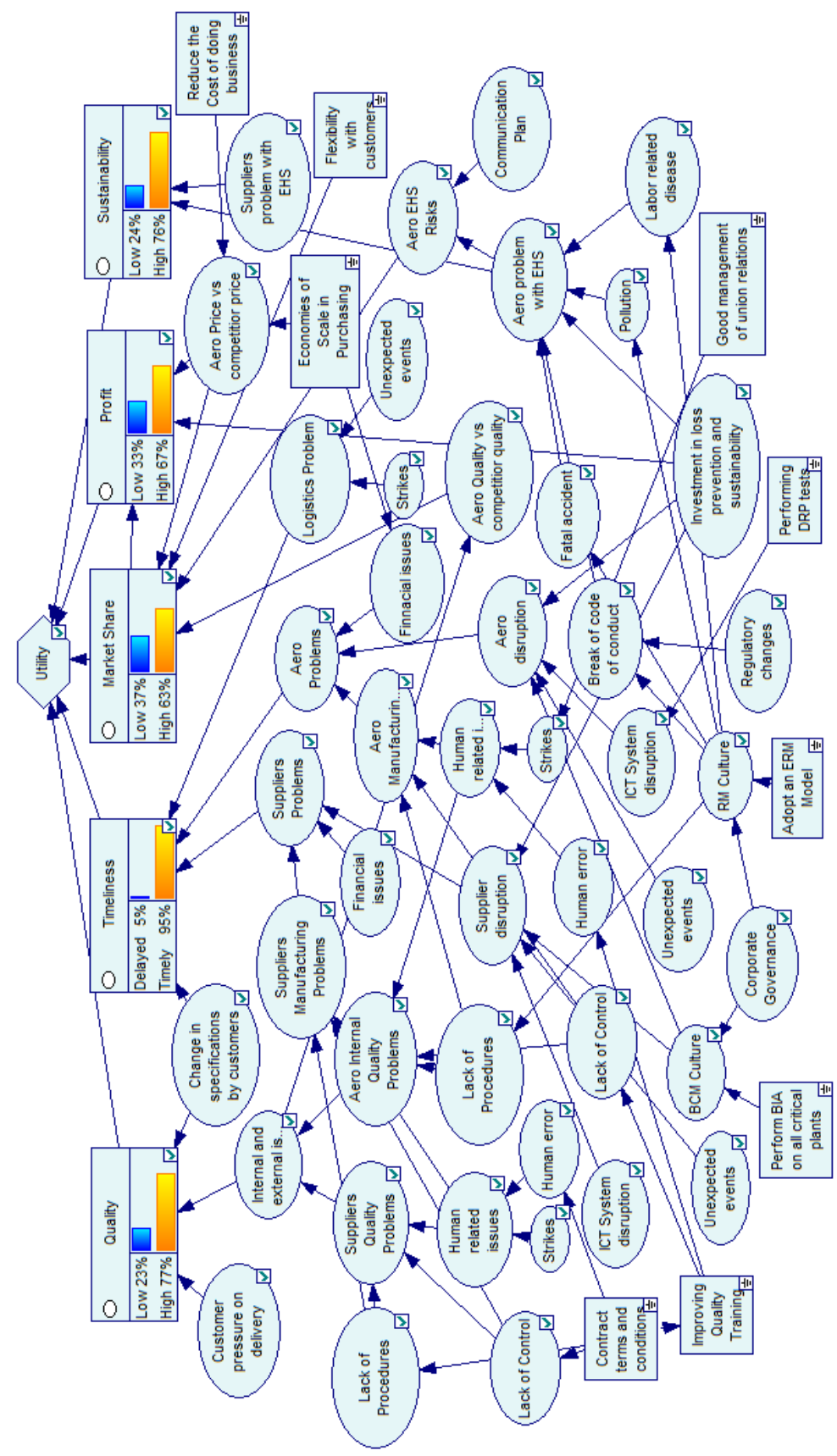

Figure A.3: Network of interacting risks, risk sources and potential strategies (GeNIe). 


\section{Appendix B. Findings on the Value and Contribution of SCRNM: Semi-Structured In- terviews}

This section is a collection of sample quotations from the interview narratives transcribed. The findings are organised into three categories: current practices of managing supply chain risks; benefits of implementing the proposed process; and the challenges involved in implementing the process.

\section{Current Practices of Managing Supply Chain Risks}

Most participants believed that there are established guidelines to conduct the risk management and generally the endeavor is to adopt the risk management standards within the enterprise. As reported in the literature, risks are classified into separate categories and assessed accordingly.

"We have standard templates which guide us what kind of topics and risks to consider during the risk management process. ... We have a tool where we nail down all kinds of risks. Cost estimation and probability are documented and signed by all stakeholders" (Resp\#6).

"We are still in a position that we don't adopt ISO 31000 standard yet. So, actually we adopt COSO (Committee of Sponsoring Organisations of the Treadway Commission) model and classify risks into two categories: financial risks and pure risks. Pure risks are subject to losses that are to some extent insurable whereas financial risks are associated with opportunities as well as downside risks" (Resp\#1).

"During risk analysis, a team composed of different team members with different roles assesses risks. The risks are considered without any correlation. Usually, given the probability and impact of risks, we try to implement mitigation plans on high risks in the risk review meeting. The risks are categorised according to the business area and categories like commercial, operational, logistics etc. are used" (Resp\#7).

A few participants acknowledged the limitation of their current practices; however, others were optimistic about the efficacy of ISO 31000 (SA, 2009) based frameworks.

"I think that the risk management process is quite mature and it serves our purpose. We started from much shallow position few years back. Project and risk management offices were introduced across the organisation to cope with the requirement of systematically managing risks" (Resp\#5).

"Since the beginning of this year, we are trying to broaden the scope of risk analysis because in silo view, we miss important things. Still we are not working on the ERM (Enterprise Risk Management) perspective. Different pieces are put together by different groups and the current risk management process does not capture the systemic interactions" (Resp\#1).

With regards to the risk treatment, it was revealed that there is no such procedure to model the trade-off between performance measures. Similarly, it is assumed that a strategy only affects a single risk and therefore, not much effort is made to assess the correlations between strategies and risks.

"During the risk review meeting, the team agrees on the selection of strategies to address the risks. Commercial issues like customer satisfaction are the main risks. The experience and knowledge of 
the team members help in selecting the best strategy to apply. For each risk, you could apply different strategies and therefore, we assess different plans" (Resp\#6).

"There is no tool available to model the trade-off involved in the objectives. For well-known risks, we take proactive approach. If we already know from our experience about the potential risks, we rely on proactive approach. However, the risk management process is more reactive. To be honest, today it is more reactive where the risks are not predictable" (Resp\#2).

"The only thing I can think of is historical trend analysis. It is not really a methodology rather it is based on historical data and best practices. There is a strong element of the experience of people. In very sizable cases, we will appoint people who have done the same thing. ... No, we do not have any such causal technique to assess strategies within the network of interacting risks" (Resp\#7).

"In terms of risk treatment, we are rude in the sense that it is the experience of your supply chain risk manager and accounts manager because in many cases, the supply chain risk manager is interested in improving the quality and the accounts manager just wants to reduce the budget. It is a negotiation between the two parties to reach an agreement. ... Rude in the sense that there is no such complex process involved in understanding the complex situation" (Resp\#6).

"We have different tools for managing risks but there is no link between the two. Like there is one tool for tracking the quality of a product. The other is for tracking the cost but there is no connection between the two. There is no evaluation of cost and effort involved in implementing a mitigation strategy" (Resp\#7).

Benefits of Implementing the Proposed Process

The participants involved in developing the model appreciated the significance of capturing interdependencies between risks and mitigation strategies.

"To start more people around the table will actually help capture holistic risks across different disciplines. If you build it for a certain project and supply chain characteristics putting much more expert knowledge, the graph would be relevant and even if new risks are introduced, the same graph can be used without starting from the scratch" (Resp\#3).

"It boils down to what happens in reality. It is all about risk management. You can only evaluate after you have gone through the (risk management) process a number of times, and experienced and recorded risks. Once the process is implemented and fully integrated within the organisation, it is more important to focus on organisational learning and this model can be helpful in monitoring risks over time and learning from the past experiences" (Resp\#5).

"I think that the process helped us develop a risk network in a very short time and it was quite helpful to think through developing the network from the performance measures. We could identify some interesting patterns and specifically the identification of sub-optimal strategies through the process is fascinating and worth investing time and effort. I am sure that such decision tools will add real value 
to any enterprise and help them make efficient investment decisions" (Resp\#1).

"It is a very interesting model where you get to know about the correlations between risks. Having something in place to provide a guideline to initial risk analysis would be a great opportunity as it can incorporate key lessons from the past in terms of the strength of dependency between risks. The most powerful thing is to take decisions. Managerial decision making is not always correct as it involves a number of biases and such tools can help the decision maker look at different facets of the problem" (Resp\#2).

"We do not have tools to model the trade-off across the objectives within an interdependent setting of interacting risks. If you do not map the main sources of risks to your performance and if you do not map the correlations then you can have a serious problem and now we are able to identify the main limitation of our current process" (Resp\#4).

"The process will really benefit from the brainstorming session involving top managers where they will be able to identify interesting patterns of risks and evaluate different strategies" (Resp\#1).

\section{Challenges involved in implementing the Process}

The main challenges were identified as the commitment to developing and updating the model, training required to enhancing the skill and knowledge of the team, the focus of established risk management standards on identifying and assessing independent categories of risks, and the resistance of the organisation to bringing the paradigm shift.

"Such type of process aimed at modelling interdependency between risks is quite expensive in terms of involving a lot of people and needing a lot of discipline. There is a cost that the company has to incur. The first challenge is to evaluate the cost and benefit analysis. The second challenge is about knowledge and how exactly you develop the competence of people involved in implementing the process. In case of a very distributed organisation like ours, it is not easy to train all the people across the organisation. The third challenge is how we capture knowledge in such project driven supply chains including risk management. It is not a simple thing" (Resp\#5).

"If we look at the ISO guide and all the standards, these describe a lot of tools for assessing risks and return on investment. Companies do not care about these correlation analyses because there is no such regulatory requirement and also, there is no literature about it from the application perspective" (Resp\#1).

"The challenge is to establish a standard tool. Checking and updating every single risk is really challenging. Secondly, usability of the tool is very important. The challenge is to get the people work and use the tool on regular basis. We have plenty of tools. We must be sure that people should be able to realise the benefit of using a new tool. It is important to demonstrate the merit of the tool so that its continuous use must be justified and guaranteed" (Resp\#7).

"Even if the process or tool is a simple one, there is always a resistance. Organisation is what it 
is so you have to play with the cards you have in your hand. The biggest obstacle is the organisation

1

2

3 charge of the risk management process" (Resp\#6). process. We need to create a steering committee or appoint a CRO (Chief Risk Officer) who is in

"It is costly in terms of time but the results are great. The challenge is in terms of eliciting values from the experts. There will be biases involved in the group decision making" (Resp\#2). 
LaTeX Source Files
Click here to download LaTeX Source Files: Latex Source Files.zip

LaTeX Source Files
Click here to download LaTeX Source Files: Latex Source Files.zip Click here to download LaTeX Source Files: Latex Source Files.zip

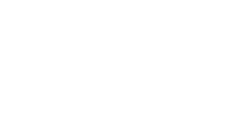
(1) (1) $\sqrt{3}$ $\sqrt{3}$ (1) (1) (1) $\sqrt{3}$ .

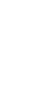
.

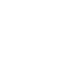
. . . . . . . . . . . . 